\title{
Review Article \\ Perspectives on the Emerging Applications of Multifaceted Biomedical Polymeric Nanomaterials
}

\author{
Ahmad Mohammed Gumel, ${ }^{1,2}$ Mohamad Suffian Mohamad Annuar, ${ }^{1}$ and Hindatu Yusuf ${ }^{1}$ \\ ${ }^{1}$ Institute of Biological Science, Faculty of Science, University of Malaya, 50603 Kuala Lumpur, Malaysia \\ ${ }^{2}$ Biotechnology Department, Faculty of Science, Federal University Dutse, PMB 7156, Dutse, Jigawa, Nigeria \\ Correspondence should be addressed to Ahmad Mohammed Gumel; gumel@um.edu.my
}

Received 26 March 2015; Revised 15 June 2015; Accepted 15 June 2015

Academic Editor: Yanlin Song

Copyright (c) 2015 Ahmad Mohammed Gumel et al. This is an open access article distributed under the Creative Commons Attribution License, which permits unrestricted use, distribution, and reproduction in any medium, provided the original work is properly cited.

\begin{abstract}
Biodegradable and biocompatible polymeric nanomaterials, serving as biomedical devices have garnered significant attention as a promising solution to therapeutic management of many chronic diseases. Despite their potentials, majority of the synthetic nanomaterials used in biomedical applications lack crucial properties, for example, ligand binding sites, responsiveness, and switchability to efficiently deliver intended drugs to the target site. Advancements in manipulating nanoscale geometry have incurred the incorporation of triggered release mechanism within the nanomaterials design. This expanded their potential applications beyond nanocarriers to theranostics exhibiting both tandem drug delivery and diagnostic capabilities. Additionally, it highlights possibilities to design nanomaterials that could translate chemical response(s) to photometric display, thus making affordable biosensors and actuators readily available for biomedical exploitation. It is anticipated that, in the near future, these implementations could be made to access some of the most difficult therapy locations, for example, blood brain barrier to provide efficient management of Alzheimer, Huntington, and other neurodegenerative diseases. This review aims to serve as a reference platform by providing the readers with the overview of the recent advancements and cutting-edge techniques employed in the production and instrumentation of such nanomaterials.
\end{abstract}

\section{Introduction}

The convergence of biotechnology, nanotechnology, and polymer science is enabling the development of new smart nanomaterials with diverse macromolecular architecture that can be utilized in biomedical applications. Among the most researched on are those that are highly responsive to the external stimuli. A wide range of polymeric nanomaterials carrying smart switchable components has been developed over the decades [1-7]. For example, multistimuli responsive nanomaterials based on amphiphilic polymer composites containing hydrophilic and lipophilic macromers with environment-sensitive micellar properties have been reported [8]. The ability of these materials to respond to the change in environmental conditions due to switchable functionality has been explored in designing several bioinspired nanomaterials. With the aim of leveraging on responsiveness properties, a number of polymeric-based nanomaterials with biomedical applications are reported, including those composed of dextran [9], polyethylene glycol [10], self-assembling peptide nanofibers [11], and biomimetic nanofibers [12].

Some of the reported applications of such materials are the delivery of biomedical therapeutics and tissue culture scaffolds [13], actuators [14], contrast aided devices in medical imaging [15], and so forth. For example, oral administration of therapeutic drugs usually resulted in low bioavailability, therefore warranting parenteral application by either intramuscular or intravenous portal. Unfortunately, most of the drugs administered via parenteral route were also reported to be rapidly eliminated from the plasma circulation $[16,17]$. For instance, adenosine, an endogenous purine nucleoside, is known to modulate several physiological processes. Undesirably, it is shown to have a plasma half-life of less than 10 seconds [18]. It is similar for norepinephrine, an important neurotransmitter, which when infused in critically ill patients results in the maintenance of arterial pressure and organ 
function [19]. Norepinephrine too was estimated to have a circulation half-life of about 2 minutes [19]. Therefore, for a maximum therapeutic efficacy to be attained, large repeated dosages of the drugs need to be administered. Consequently, the repeated or voluminous dosages often led to systemic toxicity, which is among the classic setbacks hindering the applications of major biomedical therapeutics.

Polymeric nanomaterials that are biodegradable and biocompatible have been given significant attention as a promising delivery vehicle for chemotherapy drugs [16]. Unfortunately, majority of the synthetic nanomaterials used as drug carriers lack the specific responsiveness and switchability characteristics to deliver the drugs to the target site. However, recent advancements in the fundamental understanding of biotechnological and proteomic research have led to the development of new synthetic approaches [20]. These have made possible the fabrication of stimuli responsive "smart" nanomaterials within the grasp of the biomaterials research community. Such stimuli guided drug carriers not only are capable of enclosing the drugs but most importantly help to deliver them to the specific site with a desired precision. Furthermore, the chances of systemic toxicity are significantly reduced since the protective enclosure for the drug helps to limit its direct and unwanted interaction with healthy cells [5].

For a smart nanomterial to be successfully applied in biomedical niche, the nanomaterial has to possess inherent responsiveness and switchability characteristics designed out of their unique molecular architecture and chemical interactions. Moreover, there should be sufficient flexibility to tweak the behavior of the nanomaterials to specific response(s) without compromising the carrier supramolecular ability to provide enclosure and stability to the $\operatorname{drug}(\mathrm{s})$. This review surveyed and evaluated the recent advancements in the production and instrumentation of smart polymeric nanomaterials and their composites as well as their biomedical applications. In addition, it provides the readers with some highlights on newly employed techniques in the fabrication of such nanomaterials.

\section{Biomimetic Polymeric Nanomaterials}

Nature has been inspiring material scientist in the quest of designing smart responsive polymeric nanomaterials for biomedical applications. The multifunctionality associated with nature-inspired materials allows them to be involved in, for example, elicitation of site-specific cellular responses or direct the regeneration of new tissue via biomolecular recognition. Examples of the involvement of nanomaterials in day-to-day survival of organisms are ubiquitous. For example, the nanostructures present on the surface of lotus leaf confers superhydrophobic properties to the leaves such that water droplets and dust easily roll off, thus leaving the leaf clean. Similarly, the nanometer-sized spatulae tipping the setae fibers at the tip of gecko's feet were suggested to be associated with unique reversible adhesion mechanism that holds the reptile to surfaces in a self-cleaning fashion $[21,22]$. Additionally, the fibrous cilia on the body surface of fishes and amphibians are naturally connected to sensors located at the base of the skin that detect changes in water flow conferring a superficial neuromasts sensing ability to the fish as it swims in narrow caves, dark corals grooves. It also aided the fish in sensing the presence of predators in their vicinity without the benefit of optical visibility [23]. The functionalities of these natural inspirations could be mimicked through manipulation of the supramolecular characteristics of these endowments in the process of making synthetic biomimetic materials.

The process of mimicking could be approached from ground up through the understanding of the role and function of protein sequence in the bioinspired material. This could be achieved by integrating the genotype analysis with phenotypic mapping. Unlike the conventional cDNA cloning and sequencing that takes longer time to identify the target protein sequence from the genome, a rapid generation of genotype-to-mechanical phenotype maps has been reported in this integrated approach [24]. Information gathered from this approach could enhance our understanding of subsequent structural analysis and possible applications of such materials.

Such information in combination with our knowledge on recombinant protein expression could be a handy tool in designing a bioinspired synthetic peptide that mimics the properties of the natural materials. To this end, Guerette et al. [24] have reported an accelerated design of biomimetic materials out of sucker ring teeth obtained from jumbo squid (Dosidicus gigas). In their study, the researchers circumvent the setback of time-consuming cDNA cloning by using deep sequencing RNA-seq techniques in conjunction with Raman microspectroscopy, X-ray scattering, and mechanical data to the generated genotype-to-mechanical-phenotype maps for each of these model biological materials [24]. Using their approach, the researchers were able to obtain and characterize for the first time a bioinspired protein (suckerin-39) that was melted into a biomimetic silk-like polymeric nanopillars by microwave assisted template-wetting technique (Figure 1). The nanopillar film was found to be a stable thermoplastic that can be drawn into any complex shape using facile aqueous-based processing [24]. In addition, the authors evaluated the viability and proliferation of human mesenchymal stem cells (hMSC) grown on the films. High cell viability was observed under all conditions tested, demonstrating the biocompatibility of the material. Moreover, they further reported that higher concentration of suckerin-39 protein in the casted film resulted in increasingly branched and spindleshaped cell morphologies of hMSCs, an observation that was attributed to previous reports of hMSCs lineage specification as the result of variations in matrix elasticity at different protein concentrations $[25,26]$.

Healing of peripheral nerve injuries is known to be a major challenge pertaining to nerve regeneration largely due to lack of suitable grafts techniques and poor cell penetration and repair [12]. Moreover, most of the conventional biomaterials used in tissue culture scaffold such as polycaprolactone lack the active chemical moieties on their surfaces that could support and enhance cell adhesion and regeneration. Interestingly, some extracellular matrix (ECM) proteins such as laminin were shown to exhibit a contact 

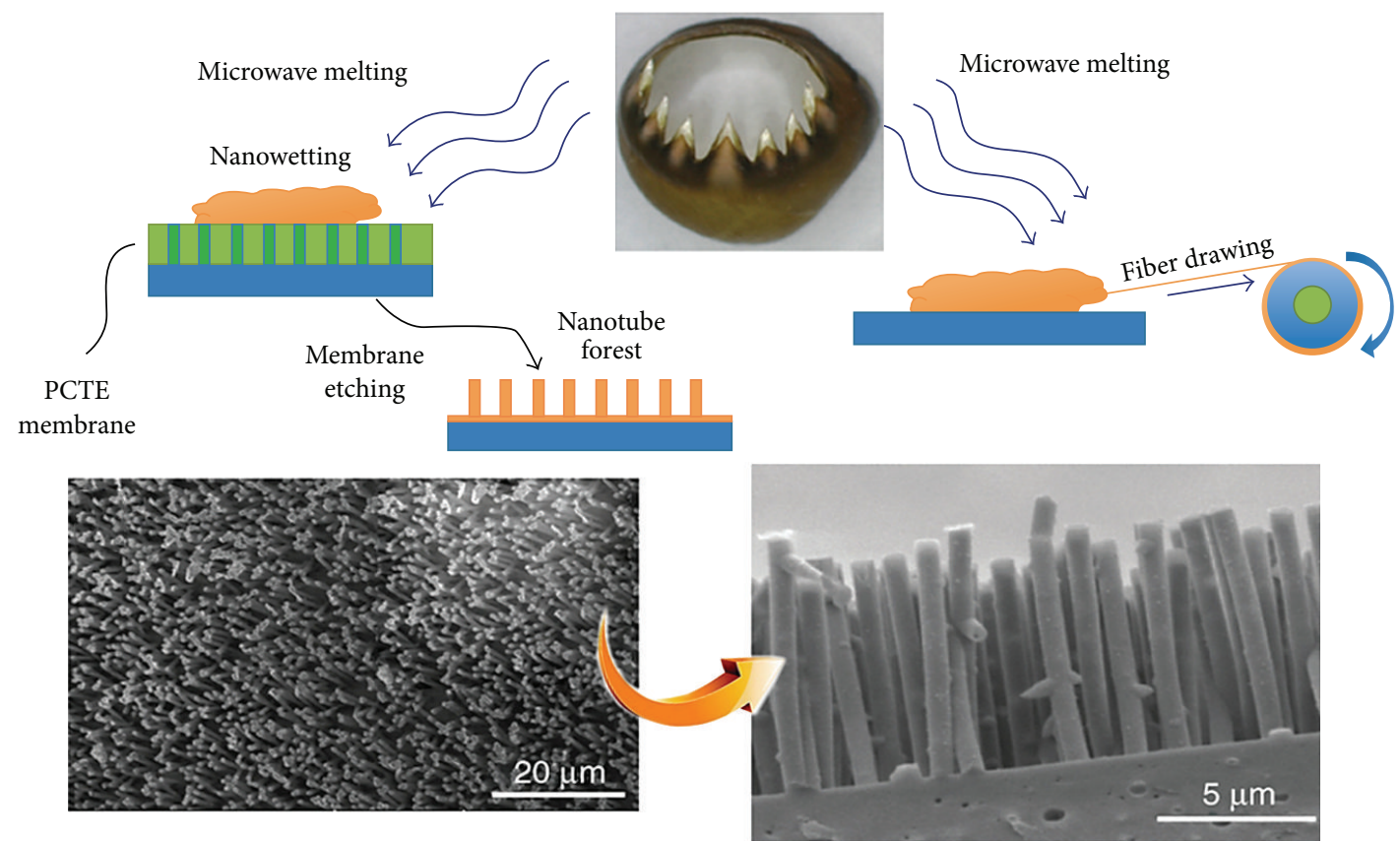

FIGURE 1: Solvent-free processing of thermoplastic suckerin-39 into nanofibers by microwave assisted template-wetting technique. Reprinted from Guerette et al. [24], with permission from Nature Publishing Group.

guidance cues that play a significant role in the regeneration of large gaps in the peripheral nerves [27]. In such kind of injuries, biomimetic nanomaterials scaffold functionalized with laminin could improve the contact property of the matrix surface, thus enhancing their regeneration capabilities. Junka et al. [12] applied carbodiimide cross-linking to attach laminin to the amine group of chitosan in electrospun nanofibers of polycaprolactone-chitosan nanocomposite (Table 1). The modified nanofibers were found to promote excellent proliferation of Schwann cells as compared to nonmodified nanofibers [12]. Due to their large surface area and excellent porosity, the functionalized nanofibers were found to mimic the structure of the native tissue fibers of the extracellular matrix. Similarly, a solution of chitosan and silk fibroin was electrospun at $18 \mathrm{kV}$ and a flow rate of $0.3 \mathrm{~mL} / \mathrm{h}$ to produce nanofibers that were immersed in ethanol/ammonia solution resulting in the change in the conformation of the silk fibroin protein [28]. An accelerated proliferation rate and osteogenic differentiation of human fetal osteoblast progenitor (hFOB) cells were observed when they were cultured on the nanofibers. Recent contribution of biomimetic electrospun nanofibers in tissue regeneration was extensively reviewed [29].

Among the reported setbacks of cancer therapy are the adverse side effects associated with the chemotherapy. Photothermal therapy based on the use of multifunctional nanomaterials with high optical absorption in the nearinfrared region is one of the promising methods in cancer treatment. The treatment is reported to generate localized hyperthermia thereby inducing irreversible damage to cancer cells in a mild way while minimizing detrimental effects to healthy tissues. Recently, Wang et al. [30] reported the synthesis of novel multifunctional phage protein-modified Au@Ag heterogeneous nanorods. The nanorods were modified by polymer based on polyanionic poly(styrenesulfonate) and were investigated for targeted optical detection and photothermal therapy towards colorectal carcinoma cells (SW620). The nanostructure was found to specifically ablate SW620 cells when exposed to $808 \mathrm{~nm}$ laser at the intensity of $4 \mathrm{~W} / \mathrm{cm}^{2}$ for 10 -minute illumination. The results indicated that the synthesized nanorods were found to be more effective agents than their gold or silver counterparts [30].

The development of robust biomimetic synthetic surface incorporating stainless property has garnered a large interest in applications such as biomedical devices, fuel transport, and architecture. Despite several decades of rigorous research, the manufacture of such materials still proves to be challenging and difficult as a result of several setbacks including high production costs, wetting by low surface tension liquid, high contact angle hysteresis resulting in poor oleophobicity, lack of self-healing property, and failure to withstand high pressure [31]. Pokroy et al. [14] used extended soft-lithography and polydimethylsiloxane as elastomeric mold to produce highly hydrophobic multifunctional, high-aspect-ratio biomimetic nanostructured surfaces based on silicon-epoxy nanoposts that could find applications as "smart" sensors, actuators, and other dynamic materials (Figure 2(a)). Building on Pokroy et al. [14] technique, Wong et al. [31] produced a selfhealing bioinspired film described as "slippery liquid-infused, porous surface" or SLIPS with pressure-stable omniphobicity (Figure 2(b)). According to the researchers, the material displayed exceptional liquid- and ice-repellency, pressure stability, and enhanced optical transparency. Thrombosis and biofouling of extracorporeal medical equipment and devices 
TABLE 1: Selected nanoparticles and their applications.

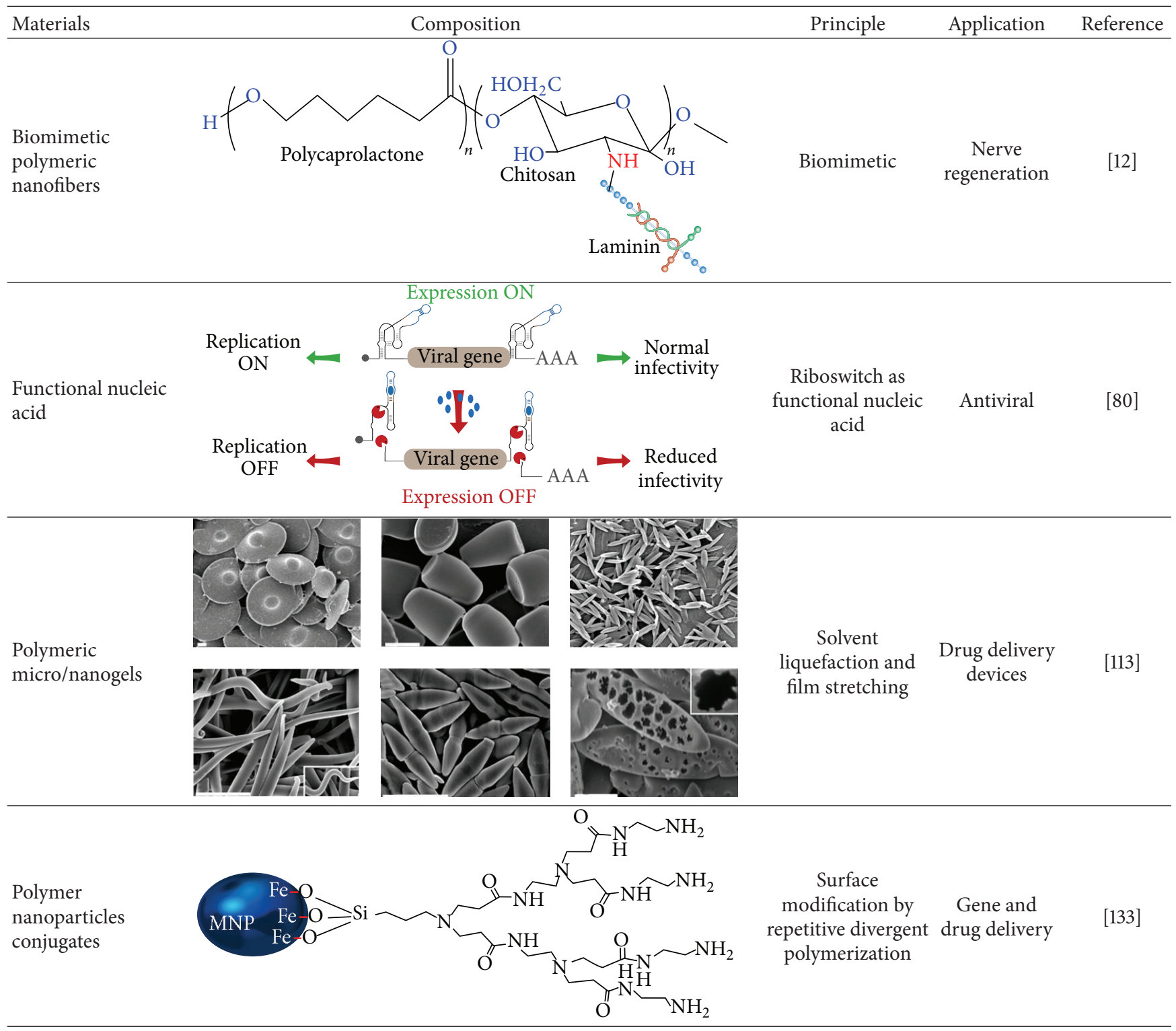

were reported to be major causes of worldwide morbidity and mortality [32]. Inspired by the stainless nature of the lotus leaves, and applying the SLIPS approach, an omniphobic nanocoating technique was described that uses tetheredliquid perfluorocarbon (TLP). The coated material was shown to highly reduce thrombosis by completely repelling blood (Figure 2(c)) and it suppresses biofilm formation when coated on surgical tubing and catheters [33].

Similarly, Hensel et al. [34], inspired by the omniphobic morphology of the springtail (Folsomia candida) skin, applied a reverse-soft-lithography and doctor-blade solution casting to produce a biomimetic super omniphobic surface based on poly(ethylene glycol) dimethacrylate. In contrast to the conventional nanopillar topology (Figure 2(a)) commonly used in biomimetic stainless materials, they came up with a material bearing honeycomb-like topology (Figure $2(\mathrm{~d})$ ). In contrast to the nanopillar structured material, the honeycomb-like material was found to exhibit high pressure resistance and excellent mechanical durability [34]. In another study, the fabrication of biomimetic stainless surface based on randomly deposited electrospun fiber mats of polymethyl methacrylate-co-fluoro polyhedral oligomeric silsesquioxane molecules was reported, which exhibited higher water contact angle $\left(>150^{\circ}\right)$ with low contact angle hysteresis [35].

Bioinspired nanomaterials were also used as biosensors. Abnormal increase in blood heptanal concentration is considered as a biomarker for lung cancer [36]. A bioelectronics sensor that mimics human olfactory system in detecting blood heptanal was reported [37]. The researchers were able to produce the biosensor by immobilizing nanovesicles containing synthetic human olfactory receptors (artificial nose) for heptanal on single-wall carbon nanotube electrode that was precoated with poly-D-lysine via charge-charge 


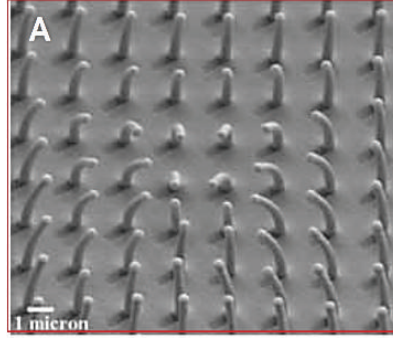

(a)
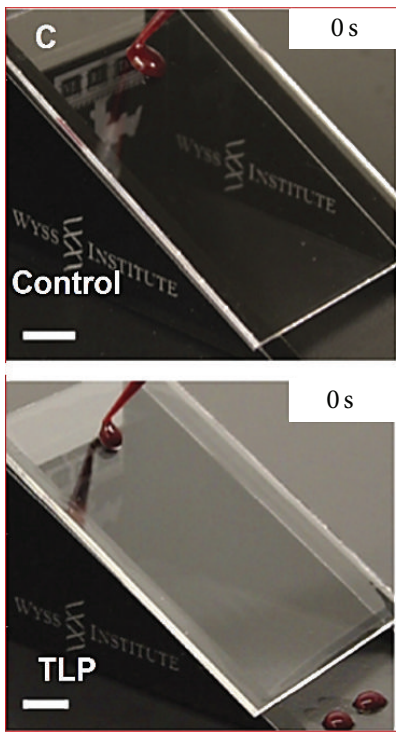

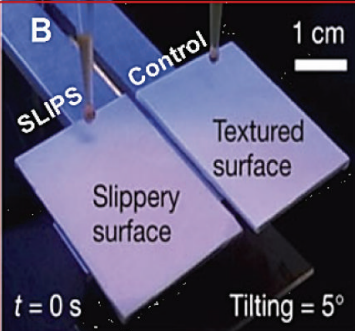

Tilting $=5^{\circ}$

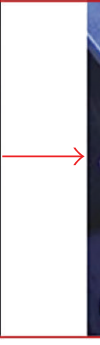

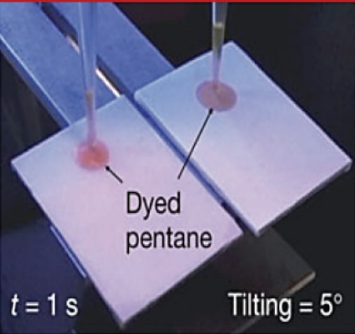

(b)
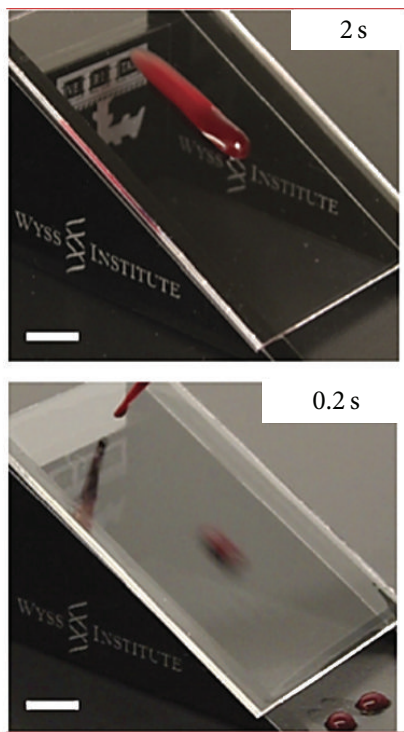

(c)
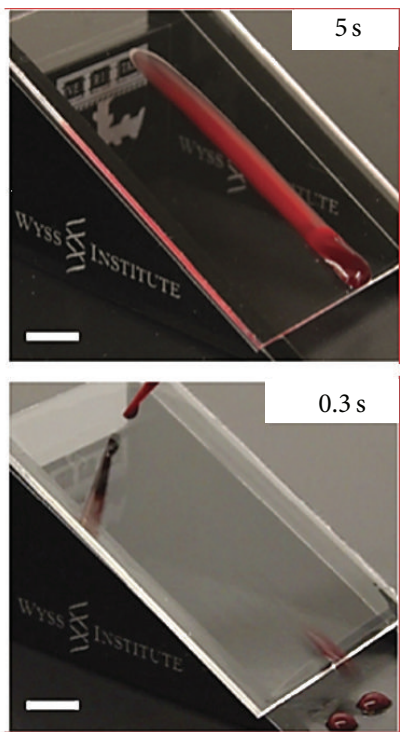

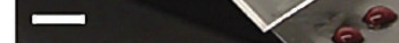
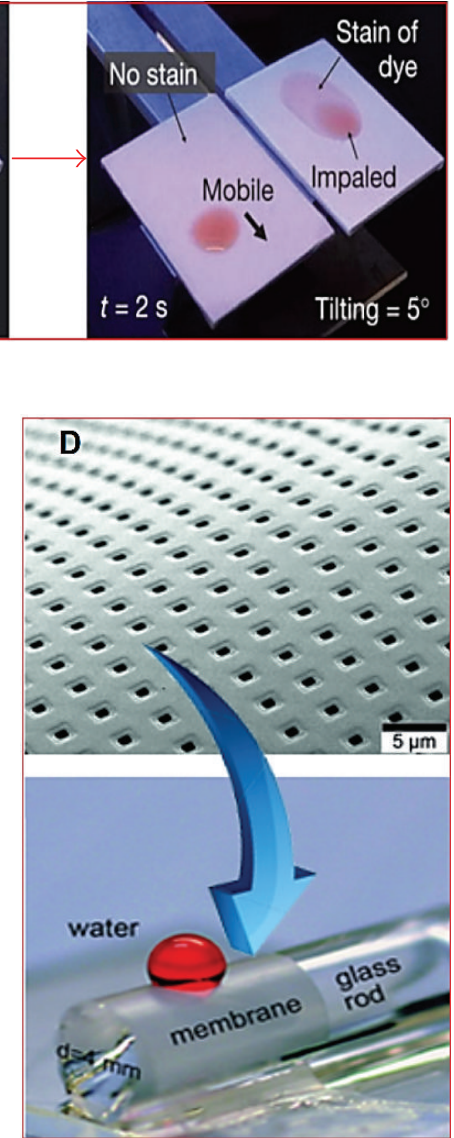

(d)

FIgURE 2: Omniphobic bioinspired nanostructured materials. (a) Scanning electron micrograph of the e-beam-actuated silicon-epoxy nanoposts. Reprinted from Pokroy et al. [14] with permission from Wiley. (b) Time-sequence images comparing mobility of pentane droplets on SLIPS and textured surface (control) adopted from Wong et al. [31], with permission from Nature Publishing Group. (c) TLP-coated surface showing blood repellency against the control without the TLP coating. Adopted from Leslie et al. [33], with permission from Nature Publishing Group. (d) Springtail-skin-inspired omniphobic polymer membrane, reprinted from Hensel et al. [34], with permission from John Wiley \& Sons.

interaction. The biomimetic sensor was found to be particularly selective towards heptanal and extremely sensitive as it can detect the heptanal even at trace concentration of $1 \times$ $10^{-14} \mathrm{M}$ [37]. Similarly, Ding et al. [38] reported a synthesis of a highly sensitive and selective biomimetic protein nanosensor based on imprinted polymers on polyethylenimine covalently bonded multiwall-carbon nanotubes. Using bovine serum albumin (BSA) as a model protein, the biosensor was found to detect BSA in the concentration range of $5.0 \times$ $10^{-7}-35.0 \times 10^{-7} \mathrm{M}$ with a detection limit of $80 \mathrm{nM}$ [38].

Color display in organisms is considered to be among a myriad of evolutionary survival strategies in nature. In some it serves as calling trait for sexual coitus, while in others it acts as defense against predators. The photonics nanostructures present on certain vividly decorated insects such as butterflies (e.g., Albulina metallica) and beetles (e.g., Hoplia coerulea) are known to produce diverse range of brilliant colors within the visible electromagnetic spectrum. It is the color diversification of such nanoarchitecture that inspired material biophysicist to produce man-made photonic nanocrystals [39-41]. Since their first introduction almost three decades ago by Yablonovitch [42], the field of artificial photonic nanocrystals research is witnessing rapid progress due to the convergence of nanotechnology, biophotonics, and materials science leading to the production of biomimetic photonic materials with a wide range of potential applications that extends beyond the biologically evolved nanoarchitectures. Indeed, it is shown that such kind of photodynamic materials has gained applications in biomedical instrumentation display technologies. Unfortunately, loss of color in brighter viewing environment has been an inevitable setback to interactive display technologies that work on spatially modulated color scheme or RGB (red, green, and blue) color filter, thus the reason behind the recent production of a working prototype of bioinspired photonic crystals full color display. The authors reported that, upon electrical actuation, the photonic-nanocrystal lattice of the fabricated display undergoes expansion and contraction, causing the photons 


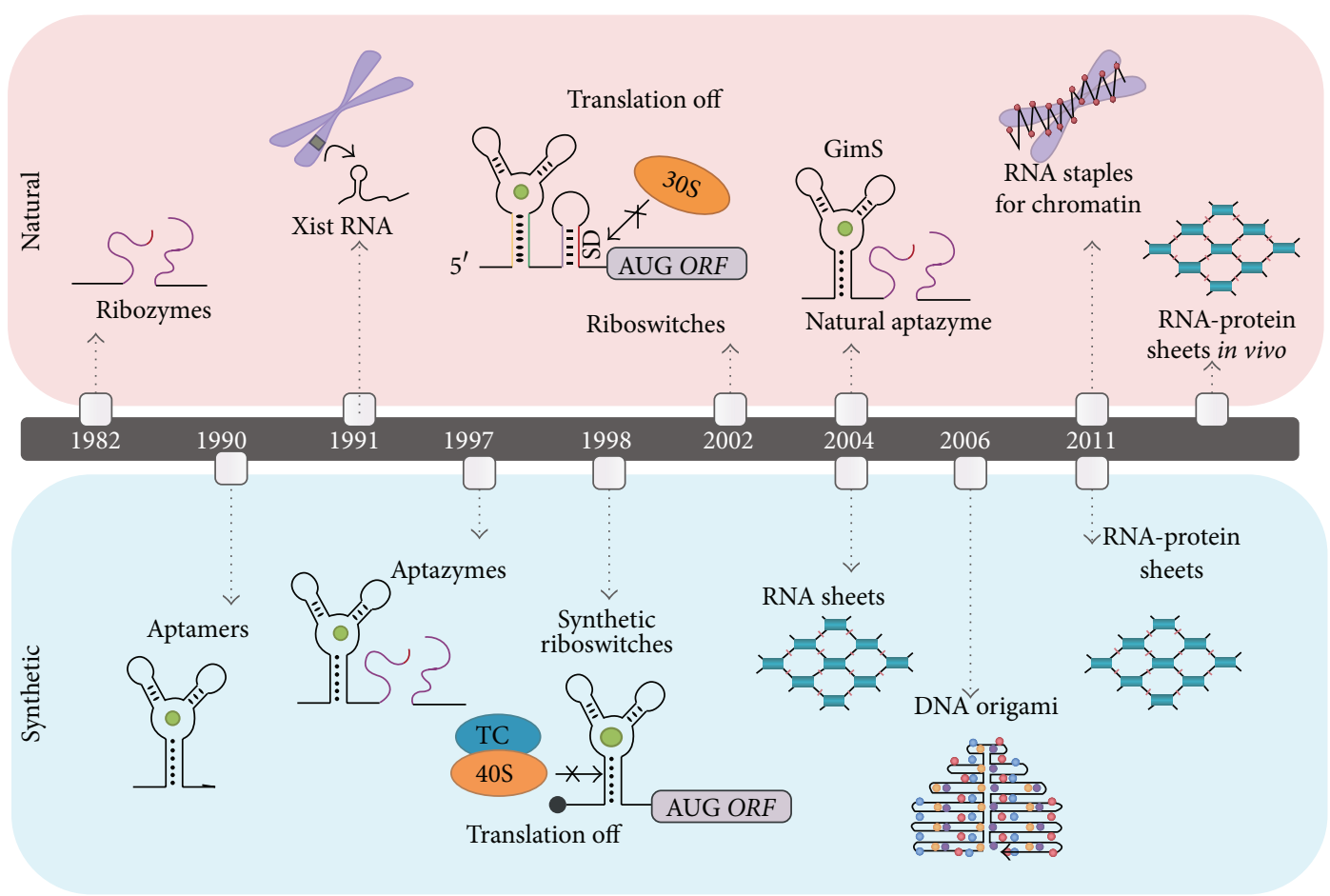

FIgURE 3: Functional nucleic acids and their applications. Adopted from [46], with permission from American Chemical Society.

to be reflected in narrow bands of wavelengths throughout the entire visible spectrum, thus resulting in displaying "nonbleachable" structural color [43].

Although biomimetic polymeric nanodevices hold promising applications ranging from target-specific delivery devices to modified biomaterials surfaces as well as biosensors, significant challenges in the development of these mimetic materials remain. This could partly be due to the milieu differences between the actual biochemical processes in vivo and those that take place in vitro. Thus, the performance of the synthetic biomimetic materials is usually found to be below expectation. However, with recent advancement in bionanotechnology, synthetic biology, and biomaterial sciences, deeper insights into the mimicry of biological principles are made possible. In near future, the emergence of highly sophisticated and more efficient biomimetic polymeric nanodevices is forestalled.

\section{Functional Nucleic Acids as Biopolymers}

The structural organization of naturally occurring biomaterials and their functions has always fascinated scientists and engineers. Almost all of these biologically occurring materials are composed of nucleic acids and protein in conjunction with other inorganic minerals. Both polypeptides and nucleic acids (NAs) are considered as major classes of naturally occurring biopolymers. To understand the principles governing the life structural organization, the structural arrangement and functional order of nucleic acids, proteins, peptides, and polypeptides are widely studied [4446]. It is now known that nucleic acids, as biopolymers, are not only restricted to genetic perpetuation since their structural composition may be functionalized to expand their utilities beyond genetic coding (Figure 3), hence referred to as functional nucleic acids (FNAs). These FNAs can be either naturally functionalized or isolated from a pool of nucleic acids library and subsequently can be functionalized in vitro $[47,48]$.

It is known that naked DNA molecules are poorly permeable to cellular membrane; they can only be transported across the membrane via the assistance of transfection agents. However, recent studies have revealed that DNAbased nanostructures are highly permeable to cell membrane. In fact, these nucleic acid nanostructures can permeate the cell membrane with high efficiency, thus the recent surge in the utilization of DNA-based nanostructures such as nanotubes, tetrahedra, and origami nanorobot as potential smart drug delivery devices [49]. Recent development on nucleic acid research has led to the designing of functional nucleic acid groups bearing binding and catalytic active sites that can function in a similar manner as much as proteins or catalyst [50, 51]. For example, functionalized DNA enzymes (DNAzymes) or RNA enzymes (Ribozymes) were reported to catalyze several reactions such as RNA cleavage, ligation, oxidation, and phosphorylation [51-53], while the functionalization of RNAs has expanded their application from mere genetic coding materials to biomimetic switches and regulators termed riboswitches [54] and riboregulators [55], respectively. Additionally, nucleic acids could be designed in such a way that they could act as receptors called aptamers. In addition to functionalized nucleic acids, bioinspired stimuli responsive polypeptides $[56,57]$ and functionalized protein 
based nanomaterials $[58,59]$ with remarkable functionalities were also reported.

Enzymes are crucial to the catalysis of biochemical reactions [60]. Although nucleic acid enzymes could be perceived as poor catalyst alternative to protein due to lack of catalytic functional groups, they are instrumental in replication, transcription, and translation of coded signals that leads to protein and peptide syntheses. The concept of nucleic acid catalysis was first reported by the demonstration of its RNAs cleaving ability [61, 62]. Since the discovery of nucleic acids enzymes, significant improvements in terms of design and attachment sequence of functional catalytic groups were made in order to enhance their catalytic function for the production of polymeric nanodevices [46]. DNAzymes offer an alternative route for controlled modification of biopolymer molecules for therapeutic applications. Addition of DNAzymes was demonstrated to enhance the performance of biosensors as they activate the amplification of the molecular beacons [63].

In conventional process, the bioavailability of lead $(\mathrm{Pb})$ poisoning is detected by a series of complex analyses such as fluorimetry, inductively coupled plasma mass spectrometry (ICP/MS), and atomic absorption spectroscopy. These analyses required multistep protocols that are time consuming [64], leading to alternative development of new assaying techniques that employ the use of calorimetric and fluorescent sensors. These methods, however, are not without considerable setbacks arising from potential false signals due to contamination with either quenchers, fluorophores, or colorants, exacerbated by time consuming protocols. The specific affinities of DNAzymes toward metallic cofactors have been exploited in designing smart biosensors that are efficient and respond rapidly to the signal. Recently, Yang et al. [65] reported the fabrication of a DNAzyme biosensor based on gold nanoparticles (GNPs) with characteristic amplified detection of $\mathrm{Pb}^{2+}$ ions. The same technique was used to develop a GNPs based biosensor using uranium metal cofactor described as Uranyl $\left(\mathrm{UO}_{2}{ }^{2+}\right)$. The sensor is shown to efficiently trace the bioavailability of uranium within the shortest possible time [66]. Furthermore, the biosensor was illustrated to work in a dual mode, that is, by "turn on" and "turn off" actions. In "turn on" mode, the presence of uranyl ions caused the dissociation of the DNAzyme from the functionalized GNPs, thus culminating in a visible chromogenic change from purple to red. On the other hand, single and double stranded DNA exhibit different absorption properties on the GNPs, and the consequence of the functionalization of these metallic nanoparticles with DNAzymes resulted in the variation of the absorption properties, hence the "turn off" action [66].

The emergence and spreading of multidrug resistance pathogens are a cause of great concern to global public health following epidemic prospect. In fact, microbial pathogens have been a cause of high morbidity and mortality rate, which is associated with huge socioeconomic burdens. Although there are several techniques at hand that are employed to test for pathogenic bacteria, most of them are expensive, time consuming, and tedious, thus presenting a significant shortcoming, particularly when it comes to timely implementation of effective preventative care in resource-limited regions. As such, there is an urgent need for cost-effective and rapid techniques for pathogen detection. Recently, a bacteria-specific RNA-cleaving DNAzyme probe was used to build a cost-effective biosensor that quickly and widely detects bacterial pathogens based on $\mathrm{pH}$ change [67]. The biosensor was built on the principle of urea hydrolysis by urease, which subsequently elevates the $\mathrm{pH}$ of the media. Urease was coupled onto the DNA template which was later attached to DNAzyme that was functionalized with magnetic beads (Figure 4(a)). Hence, using $\mathrm{pH}$ sensitive dye or paper, the presence of pathogenic bacteria in the sample is detected (Figure 4(b)). Similarly, split G-quadruplex DNAzyme probes were used to develop an efficient biosensor that allows for Mycobacterium and Salmonella rapid detection [68]. Likewise, DNAzyme based catalytic molecular logic gate was developed [69]. In this type of DNA-based logic-gates, the catalysis is controlled via toehold-mediated DNA strand displacement reaction. The technique could be potentially employed for in vivo amplified detection of viruses and other pathogens [69].

In contrast to DNAzymes, the ribozymes are reported to catalyze self-scission of the RNA backbone by a mechanism similar to RNase. From structural morphology studies, Cochrane and Strobel [51] reported that naturally occurring ribozymes are grouped into five main subtypes, namely, hammerhead, hepatitis delta virus (HDV), glmS, Varkud satellite (VS), and hair-pin ribozymes. Series of allosteric ribozymes capable of sensing bacterial second messenger cyclic diguanosyl- $5^{\prime}$-monophosphate (c-di-GMP) were created in vitro [70]. In their study, the researchers designed the allosteric sensors by using nanobridges of random sequence to join a hammerhead self-cleaving ribozyme with an aptamer from the c-di-GMP riboswitch. The developed allosteric biosensor was reported to sense the cytoplasmic concentrations of c-di-GMP as low as $90 \mathrm{nM}$ [70]. The study highlighted that such system could be potentially employed in the screening for pathological bacterial infection in samples obtained from complex biological system. In another study, vaginal installation of nanovesicles loaded with small interference RNA (siRNA) was reported to bring about the silencing of endogenous genes in the genital tract, thus conferring protection against infectious disease [71]. Nanoparticles of FNAs have also found application in the management of human cytomegalovirus (HCMV) infection, a major cause of morbidity and mortality in immunocompromised individuals and infants. Genetically, UL49 is a highly conserved gene of HCMV and its concentration is tantamount to the presence of the virions. Thus, nanoparticles of ribozymes based on siRNA were evaluated in inhibiting HCMV infection by targeting and silencing UL49 gene [72]. The result demonstrated a significant reduction in the virions after the test, suggestive of its potential therapeutics implication [72]. Correspondingly, nucleic acids therapies have opened a new horizon and optimism for curing HIV by inhibiting the virions replication. In the pathogenesis of human immunodeficiency virus type 1 (HIV1), Gag protein is highly significant in virions replication 


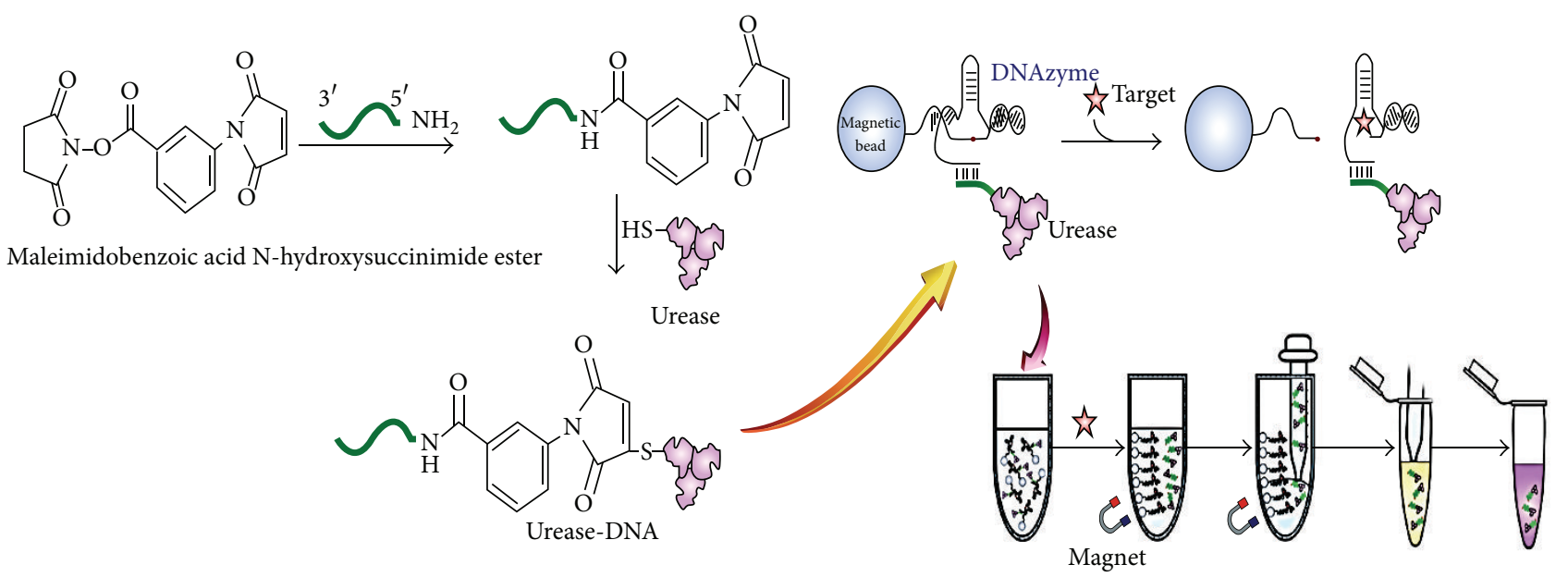

(a)
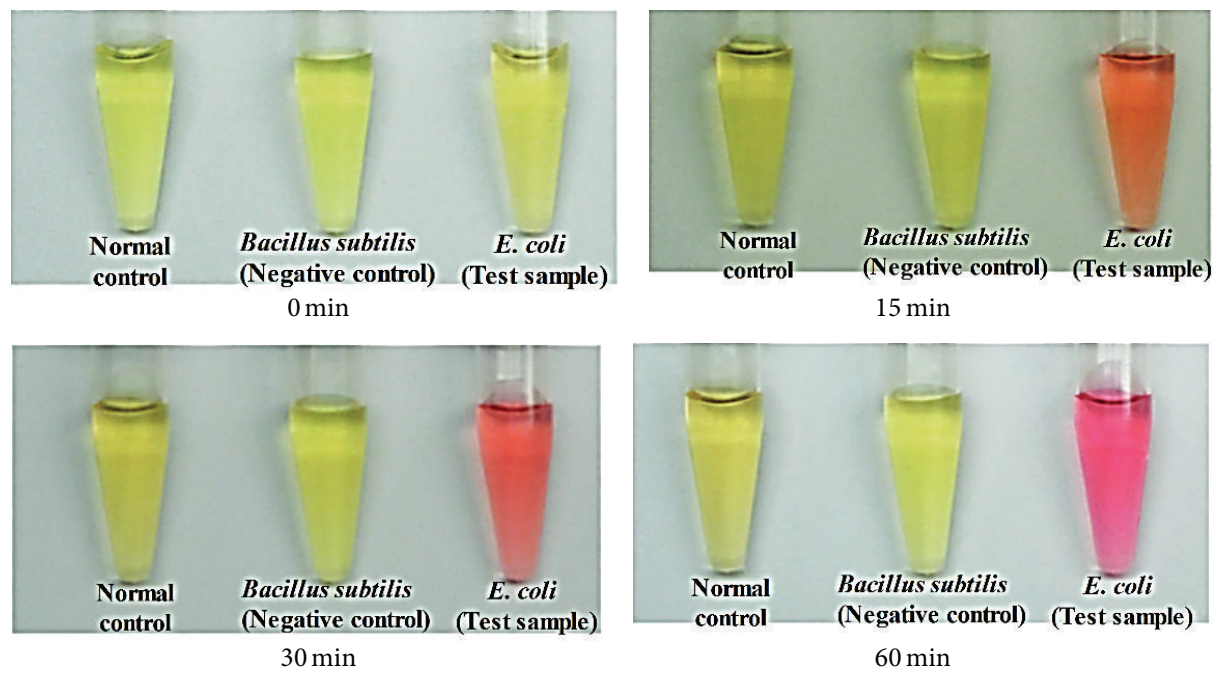

(b)

FIGURE 4: Schematic representation of DNAzyme pH-sensitive biosensor for the detection of pathogenic bacteria. Reprinted from Tram et al. [67], with permission from John Wiley \& Sons.

[73]. In fact, Gag protein is considered to be responsible for initiating the assembly and release of HIV-1 particles [74]. Previously, synthetic hammerhead ribozymes engineered to target HIV-1 Gag transcript were shown to inhibit HIV in vitro [75]. Additionally, human cells engineered to express the hammerhead ribozymes that target HIV-1 Gag protein showed a significant reduction in the level of HIV antigen p24, indicating a promising therapeutic efficacy against HIV [75]. Previous review has expounded on the clinical relevance of ribozymes in targeting HIV virions [76]. Human epidermal growth factor receptor 2 (HER-2) is a protooncogene known to encode transmembrane receptor protein $p 185$, which is associated with the growth and metastasis of adenocarcinoma [77]. The HER-2 is expressed in about $30 \%$ of human breast cancer. Two anti-HER-2 ribozymes that specifically target HER-2 mRNA were synthesized and separately assembled in nonviral eukaryotic expression vector. The engineered nanovectors were subsequently used to transfect malignant breast cancer cell lines MCF-7 [78]. The study demonstrated a remarkable downregulation of HER-2 mRNA and concomitantly $p 185$ protein in ribozymes transfected MCF-7 cell lines in addition to upregulation of oncosuppressor gene $p 53$ [78].

The ability of RNA to fold into 3D structures with pockets and cavities is a strong indication to its potential as binding ligands [79]. This leads to the development of RNA based bioswitches described as Riboswitches. These switches are a short sequence of RNA that depend on ligand modulation coupling and function as biosensor. They exhibit the capability of regulating gene expression by $\mathrm{ON}$ - and OFF-mechanism in response to changes within their milieu. Naturally, riboswitches are found in untranslated region of mRNA [77]. Recently, artificial riboswitches described as "aptazymes" were shown to inhibit replication in adenovirus and measles by knocking down the adenoviral DNA and measles RNA, respectively, hence a potential cure for these infections [80]. The artificial riboswitch was designed by 
linking a self-cleaving ribozyme sequence with a domain of ligand-binding aptamer (Table 1). The ON- or OFFswitching feature was achieved through the modification of the aptamer-ribozyme linking sequences [80]. Earlier review has discussed the application of riboswitches as antimicrobial agents via gene regulations [77].

Elastin is another extracellular matrix protein present in the connective tissues of vertebrates. The elasticity and resilience of muscles and tissues are attributed to elastin. Structurally, it is composed of several cross-linked amino acids particularly Valine, Alanine, Lysine, Proline, and Glycine, built as repeated units of tetramer, pentamer, or hexamer of these amino acids [81]. A polymeric derivative of elastin amino acids in combination with random guest residue of any amino acid $\left(X_{\text {amino }}\right)$ other than Proline [poly-

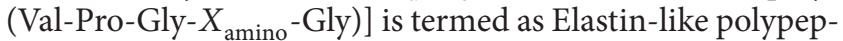
tides (ELPs). The process of functionalizing target protein to yield such kind of biopolymer is called ELPylation [81]. Interestingly, the striking chemical similarity of ELPs to natural elastin makes them invulnerable to immunologic rejection, thus their biocompatibility [81]. Being biocompatible and negated of immunogenic toxicity, polypeptides such as ELPs have garnered a lot of interest in biomedical applications [57, 81-84].

ELPs based nanocarriers were used as controlled released devices. The control release of various substances such as antibiotics and contraceptives and the modulation of their release have been a subject of intense research. The modulation and control release of these compounds are essential in order to ensure target specific delivery, accurate dosage for maximum therapeutic efficacy, reduced toxicity, and improved patience convenience. For example, in diabetic patients, shots of insulin are required to maintain the normal blood glucose level. In such a case, controlled release of insulin is highly desirable, especially to patients with no immediate access to health care facilities. ELPs nanovesicles were used as a system to effect controlled release of insulin and other therapeutic drugs [85]. Similar controlled release modulation was employed in multiple hormone therapy [86]. Similarly, in ELPs design, the lower critical solution temperature (LCST) can be modulated by manipulating the type and distribution of the guest amino acid residue, polymeric molecular weight $\left(M_{W}\right)$, and solution parameters such as $\mathrm{pH}$ and ionic strength as well as the polymer concentration [87]. Thus, bearing that in mind, highly specialized ELPs based drug carriers with precise controlled release were designed [58, 88-90]. Colorectal cancer and other intestinal injuries were shown to affect myriads of individuals worldwide. Surgical anastomosis is commonly employed in such kind of intestinal and colorectal surgery. Unfortunately, this kind of procedure is known to cause infection that could lead to life-threatening consequences. Recently, a mild and less invasive procedure was developed. In the procedure, plasmonic polypeptide nanocomposite solders that were prepared based on ELPs cross-linked with gold nanorods were used to weld ruptured intestinal tissue upon exposure to near-infrared (NIR) laser irradiation [91]. Self-assembled recombinant ELPs nanoparticles derived from engineered tropoelastin monomers that have hydrophobic and hydrophilic amino acid residues were reported [92]. The amphiphilic property of the polypeptide warrants its micellar coacervation behavior, resulting in their assembly into fibrillar matrices possessing solubility and mechanical properties similar to those of natural elastin [92]. Recently, ELPs made up of double-hydrophobic block copolypeptides based on prolinerich [poly(VPGXG)] and glycine-rich [poly(VGGVG)] were synthesized [93]. The synthesized polypeptide was found to reorient itself at higher temperature resulting in distinct structure of $\beta$-turns and $\beta$-sheets, respectively (Figure 5 ). For example, the block polypeptide in water at $45^{\circ} \mathrm{C}$ was observed to assemble into nanoparticles of mostly $\beta$-turns structure and thereafter connect to one another by $\beta$-sheets structure resulting in nanofibers (Figure 5). Bioinspired novel materials such as these promised considerable potentials in tissue engineering and drug delivery.

In an attempt to avoid chemical cross-linking in ELPs synthesis, enzymatically cross-linked ELPs were constructed using transglutaminase ( $t \mathrm{TG}$ ), an enzyme that catalyzes a calcium-dependent acyl transfer reaction between peptidebound glutamine residues and various primary amines, resulting in the formation of $\gamma$-glutamyl- $\varepsilon$-lysyl covalent bond $[94,95]$. In a similar study, $t$ TG was used as a biocatalyst to cross-link a smart polypeptide containing lysine and glutamine as guest residues forming nanovesicles that encapsulate chondrocytes [95]. The synthesized nanovesicles were found to enhance extracellular matrix deposition and cellular viability. This advantageous position is attributed to the presence of $t$ TG implied to have improved the organization and stability of extracellular matrix and enhance cell growth and differentiation [87]. Alternatively, lysyl oxidase is known to oxidatively deaminate lysine residues resulting in covalent intermolecular cross-linking into a stable elastin matrix [95]. The cross-linking process followed spontaneous condensation reaction, which led to the formation of dehydrolysinonorleucine and allysine aldol that condensed to form the predominant cross-links of insoluble elastin described as desmosine and isodesmosine [95]. The covalently linked elastin polymeric matrix was observed to be highly insoluble in most solvents except those that solvate peptides. In fact, this specific elastin matrix was reported to withstand strong alkali $(0.1 \mathrm{M} \mathrm{NaOH})$ digestion [95]. Previous studies have revealed the use of lysyl oxidase to synthesize ELPs based on recombinant tropoelastin into nanovesicles capable of coacervation [96]. Similarly, the mechanical strength of ELPs was reported to be improved by cross-linking with a hydrazone [97].

The severe systemic toxicity and suboptimal pharmacokinetics associated with several cancer therapeutics are reported to be among the limitations for their maximum efficacy [87]. Recently, amphiphilic anionic copolymer of polypeptide and methoxypoly(ethylene glycol) with three functionalized domains is synthesized and used as a nanocarrier for the delivery of cationic anticancer drug doxorubicin hydrochloride [98]. The study demonstrated that encapsulating the doxorubicin (DOX) in the polypeptide-based nanovesicles resulted in an increased inhibition of cancer cell viability. Similarly, the semicrystalline property of silk in combination with the elastomeric feature of elastin was 


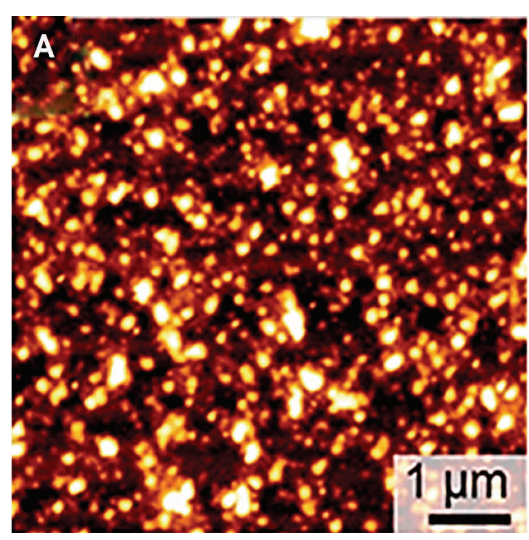

(a)

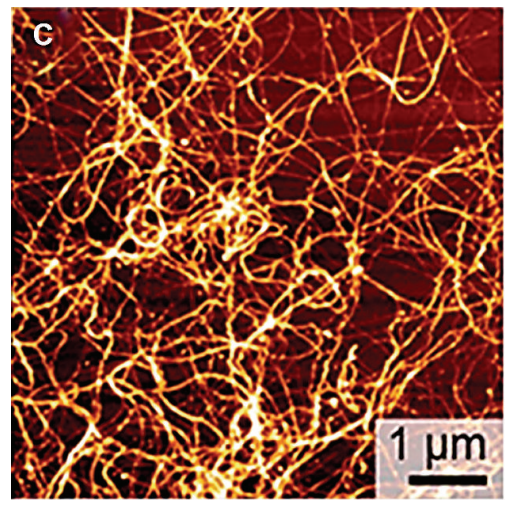

(c)

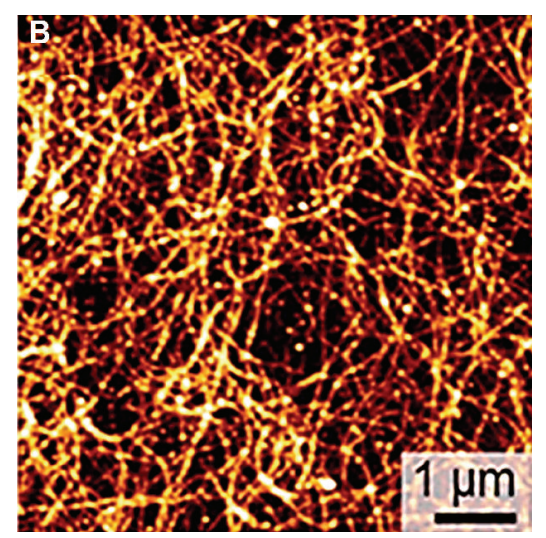

(b)

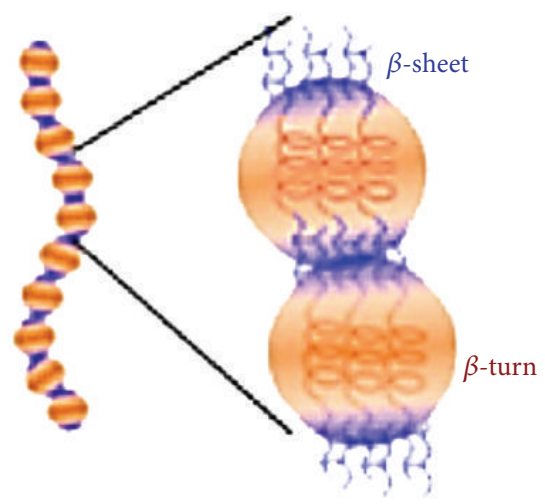

(d)

FIGURE 5: Self-assembled block polypeptides in water at $45^{\circ} \mathrm{C}$ : (a) nanoparticles formation 1 day after fabrication. (b) Day 2 of fabrication showed nanofibers formation through $\beta$-sheets linkage. (c) Seven days in water showed extensive network of nanofibers. Adopted from Le et al. [93], permission from American Chemical Society.

exploited to fabricate a silk-elastin-like protein (SELPs) composites biopolymer with tunable self-assembly behavior that was used to form nanovesicles for a controlled-delivery of cancer therapeutics [99]. An overview on the application of SELPs as therapeutics nanocarriers was published by Huang et al. [100].

In another study reported by Huang et al. [101], the primary $\mathrm{NH}_{2}$ groups of cationized functionalized BSA were coupled with mercaptothiazoline-activated pNIPAm polymer chains which were used as modules to produce conjugated protein-polymer nanovesicles (proteinosomes) that serve as a drug carrier device (Figure 6). In their study, the researchers found that carbodiimide activation using 1,6-hexanediamine in cationization of aspartic and glutamic acids enhances the number of accessible amine groups available for activated polymer coupling and cross-linking. Interestingly, they also observed that coupling the activated pNIPAm with native BSA (nonfunctionalized) showed a marked reduction in the yield of polymer-protein conjugates. They further demonstrated that the vesicular diameter could be controlled within a narrow range $(10-100 \mu \mathrm{m})$ by changing the loading concentration of conjugated polymer/protein building blocks while maintaining a constant volume fraction $\left(\varphi_{w}\right)$ of the aqueous/oil mixture [101].
The focal importance and application of FNAs have been researched extensively. Some have found application in biosensors, multiple hormone therapy, and downregulation of cancer genes as well as drug carrier devices. Although the SiRNA were reported to inhibit the HCMV genes, the point at which the gene is needed for viral replication needs to be unraveled. Additionally, an urgent need of additional research is a need to check the effectiveness of the antiHIV aptamers in vivo. Likewise, the preclinical assessment of these promising materials and techniques ought to be a researchable field.

\section{Biomedical Polymeric Nanogel}

The high water absorption capability, biocompatibility, size adjustability, diverse physicochemical structures, and large 3D surface area for multivalent bioconjugation made polymeric nanogel a potential candidate for biomedical applications. For example, the spontaneous changes with temporal periodicity, such as cell cycles, biorhythms, heartbeat, brain waves, and hormones secretion, are described as living system autonomous oscillation. Inspired by this, BelousovZhabotinsky (BZ) reaction was applied to prepare a novel biomimetic polymer nanogel that autonomously oscillates 


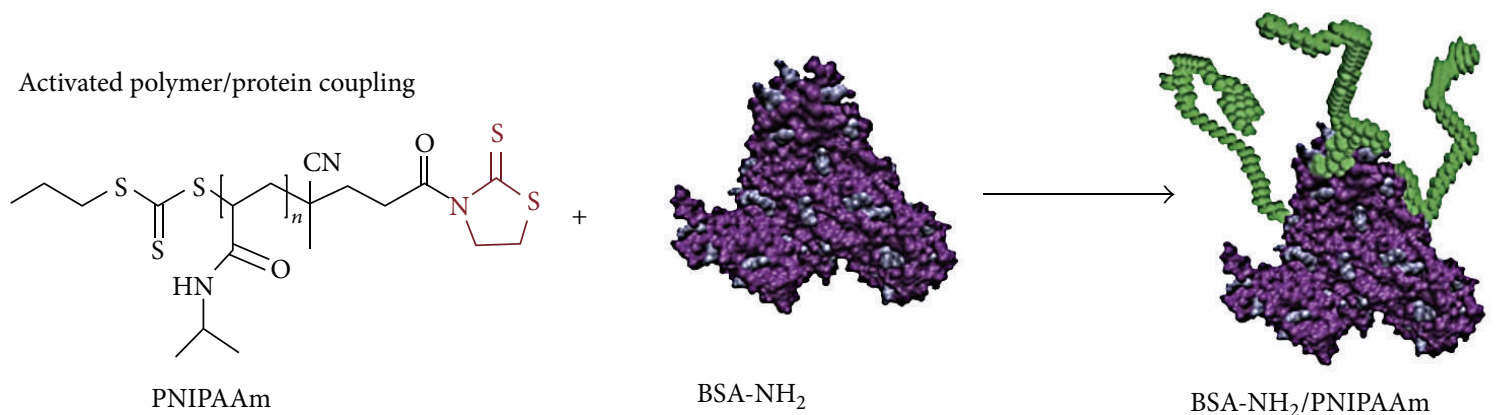

Nanovesicles assembly
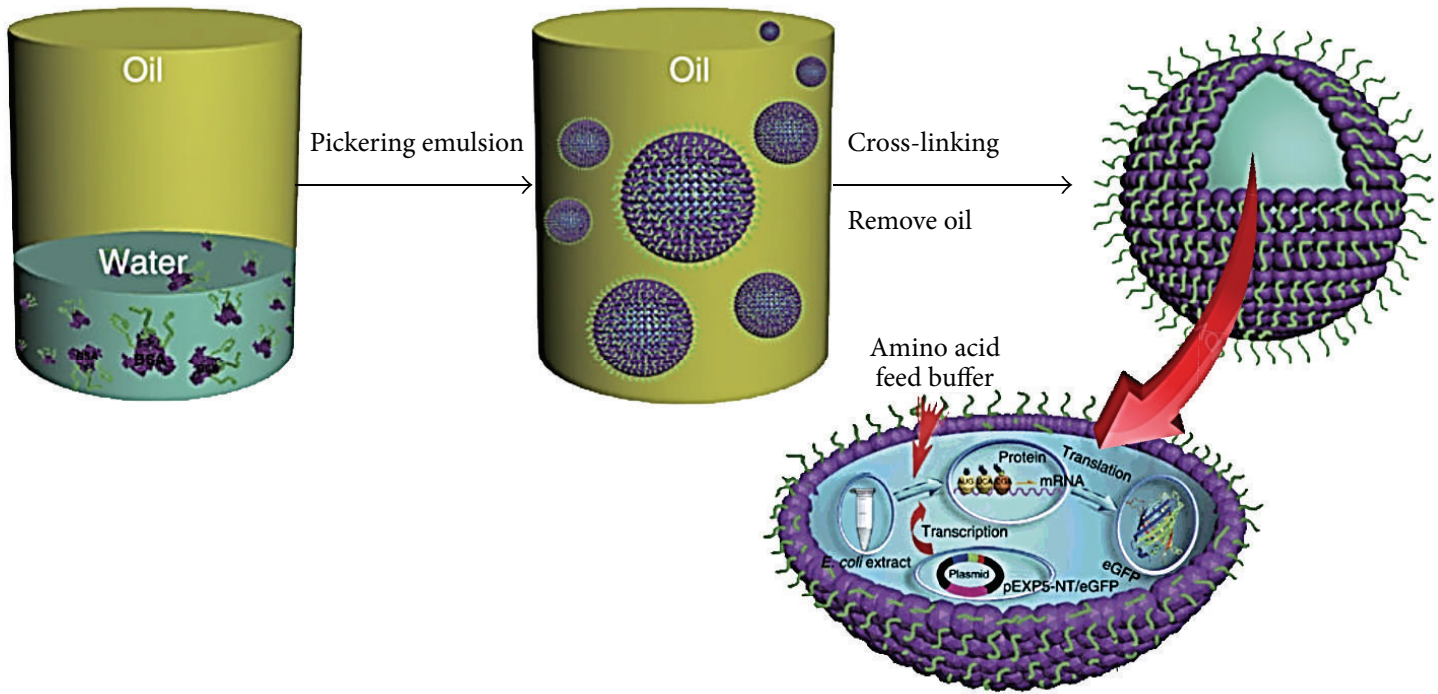

FIGURE 6: Schematic diagram of smart drug delivery proteinosomes based on protein/polymer nanovesicles capable of cell-free gene expression of eGFP. Adopted from Huang et al. [101], with permission from Nature Publishing Group.

without an external control or switch [102]. In another study, following the same BZ reaction, surfactant-free precipitation polymerization method was employed to fabricate submicron-sized self-oscillating microgel beads based on thermosensitive poly-N-isopropylacrylamide (pNIPAm) macromer [103] that could expediently be applied as bioactuators.

Similarly, in the search for effective biomedical actuators, thermoresponsive nanogels exhibiting lower critical solution temperatures (LCSTs) in aqueous media have received much attention [104]. Previously, reversible addition-fragmentation transfer (RAFT) polymerization in water was used to polymerize NIPAm in presence of sulfanylthiocarbonylsulfanyl (STS) groups as controllers [105]. The synthesized PNIPAm was grafted onto pullulan via self-association resulting in nanogels with $40-50 \mathrm{~nm}$ diameter. The nanogels STS groups were subsequently converted to thiol by isopropylamine as an oxidant, hence giving rise to chemically cross-linked thiol terminated nanogels with dual redox and temperature responsiveness [105]. Chen et al. [106] reported the use of host-guest chemistry to fabricate dual stimuli responsive supramolecular polypeptide based hydrogels that were tested as drug delivery vesicles. Additionally, the authors elaborated that the copolymer topology and the polypeptide composition are primary in tuning the hydrogel's responsiveness (i.e., temperature and $\mathrm{pH}$ ) and its mechanical and controlled drug release properties.

Additionally, nanogel responsive to both $\mathrm{pH}$ and ionic strength and temperature was synthesized using a polymeric core of poly[2-(N,N-diethylamino)ethyl methacrylate] and tethered chains of polyethylene glycol functionalized with carboxylic acid groups for biotagging [107]. The research showed the nanogel to have potential biomedical applications as endosomolytic agents for nonviral gene delivery, drug delivery carrier, nanoreactor, and skinspecific nanocatalyst for reactive oxygen species [107]. In another study, a pulsed drug delivery was achieved in self-rupturing nanogels (Figure 7) composed of degradable dextran-hydroxyethyl methacrylate with the polyelectrolytes poly(allylamine hydrochloride) and sodium poly(styrene sulfonate) prepared using layer-by-layer deposition [108]. The hydrogel was able to deliver the drug via pulsating motion due to the increase in swelling pressure to a critical point, consequently resulting in the rupturing of the polyelectrolytes core shell to release the drug.

The effectiveness of small interference RNA (siRNA) therapy largely depends on the successful delivery of the RNA to the target site. In an attempt to improve siRNA 


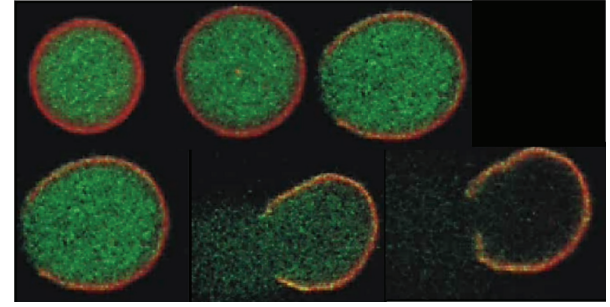

FIGURE 7: Self-ruptured hydrogel for pulsed drug delivery. Adopted from De Geest et al. [108] with permission from John Wiley and Sons.

delivery, inverse emulsion polymerization based on electrostatic interaction was used to produce nanogels that effectively entrapped siRNA in high loading capacity [109]. Based on confocal microscopy study, the siRNA-loaded nanogels were taken in by human hepatoma cell (HuH-7) even at high loading without significant toxicity. In another study, Agarwal et al. [110] studied the effect of nanogel particle geometry on the cellular uptake in drug delivery application. The findings showed that nanogel uptake is dependent upon particle concentration, cell-particle adhesion, and strain energy for membrane wrapping around the particle. These effects are known to be particle-shape-dependent [110]. Cellular tumor antigen protein $(p 53)$ is a protein encoded by gene TP53 in humans. It regulates cytogenesis and thus suppresses tumor. Recently, biocompatible thermosensitive cationic nanogel was shown to effectively deliver $p 53$ gene to gastric tumor (N87) cells consequently inhibiting the tumor growth [111]. The study demonstrated that the physical properties of the nanogels influenced their in vivo transport ascribed to stable polyplex structure, small size, narrow size distribution, and weak surface potential [111]. In an attempt to improve the in vivo transport of nanogel carriers, stepand-flash nanoimprint lithography was used to fabricate enzyme-triggered shape-specific nanogels as small as $50 \mathrm{~nm}$ and was tested for the delivery of antibodies and nucleic acids [112]. Silicon wafer was used as a substrate support to fabricate the nanogels in various sizes and shapes. Equimolar mixture of polyethylene glycol diacrylate (at 75\% w/w) and peptide oligomer (Gly-Phe-Leu-Gly-Lys) was used to create a cross-linked nanogel matrix that was harvested directly into aqueous buffers using a biocompatible, one-step release technique. Upon the addition of cathepsin B enzyme, the nanogels showed improved drug release characteristics [112]. Alongside lithography imprint, several techniques of producing nanogels of different particle sizes and shapes were exploited. Champion et al. [113] employed the use of heat or solvent liquefaction followed by film stretching to give nanoparticles with different sizes and shapes (Table 1).

Despite the promising anticancer therapeutic potential, they faced a debilitating setback in the form of low availability that reached the Langerhans cells presumably due to diffusion limitation as they failed to diffuse through the muscles when administered via transdermal injection [114]. Iontophoresis via an application of small electric current was employed to efficiently deliver cancer antigen $g p-100$ peptide encapsulated in nanogel for anticancer therapy [114]. The process resulted in the accumulation of $g p-100$ peptide loaded nanogels in the epidermis and subsequent suppression of the tumor growth.

Bacterial infection caused by Streptococcus pneumoniae includes otitis media, pneumonia, bacteremia, and meningitis in both children and adults. Recently, Fukuyama et al. [115] developed nanosized nasal vaccine delivery system using a cationic cholesteryl group-bearing pullulan ( $c \mathrm{CHP}$ ) nanogel containing pneumococcal surface protein A (PspA). The research demonstrated the safety and efficacy of the developed PspA-loaded $c \mathrm{CHP}$ on central nervous system in the course of pneumococcal infection in nonhuman primates. In comparison to the administration of naked PspA alone, the study indicated a longer retention of florescent-labelled $P s p A$-nanogel in nasal cavity when administered to rhesus macaques (Macaca mulatta). Furthermore, the study noted that, using positron emission tomography (PET) in conjunction with real-time imaging, no deposition of the florescentlabeled $P s p A$ was seen in the olfactory bulbs or cerebrum (Figure 8), supporting the safety of the delivery method. A stimuli-response to near infrared (NIR) light triggered drug delivery system based on amphiphilic chitosan-coated single-wall carbon nanotubes (CNT) encapsulated in the thermo-pH responsive nanogel (CS/PNIPAAm@CNT) has been developed to enhance the transportation and delivery of tumor-targeting drug doxorubicin (Figure 9) and anticipated to minimize the severe side effects of chemotherapy [116]. The prepared nanogels were found to exhibit high doxorubicin (DOX) loading capacity $(\sim 43 \%)$. The observation was attributed to the occasion of Schiff-base formation between chitosan and DOX as well as $\pi-\pi$ stacking and hydrophobic interaction between the substrates [116]. In order to improve the in vivo application of the fabricated nanogels, the researchers experimented on the addition of polyethylene glycol diacrylate $\left(M_{w}=250 \mathrm{Da}\right)$ to tune the lower critical solution temperature of the nanogel to an almost physiological temperature of about $38^{\circ} \mathrm{C}$ [116]. Additionally, using confocal laser scanning microscopy, the researchers were able to demonstrate the effectiveness of the NIR irradiation in triggering the DOX release from the nanogel, thus causing a significant level of cytotoxicity to HeLa cells [116]. In a similar study, a combination of enzymatic transesterification and emulsion copolymerization was employed to prepare poly-galactose-functionalized multiresponsive nanogels for hepatoma-targeted drug delivery [117]. Similar to this study too, Qin et al. [116] employed the use of confocal laser scanning microscopy and flow cytometry to establish the elevated cellular uptake of DOX encapsulated in the functionalized nanogels.

The manipulation of nanoscale geometry and the incorporation of triggered release mechanism within the nanogel design opened up a new frontier in drug delivery system. Utilizing nucleic acids probe coupled to the nanogels, it is now possible to design the nanogels based on diseasesensitive release triggers. Most importantly, it is encouraging the urge in advanced research pertaining to the nanoparticle size and shapes, which allows for flexibility in leveraging nanogels designs for different forms and properties. This is anticipated to improve significantly drug loading capacity 
$\# 1^{\mathrm{a}}\left(\left[{ }^{18} \mathrm{~F}\right] P s p A\right.$-nanogel $)$
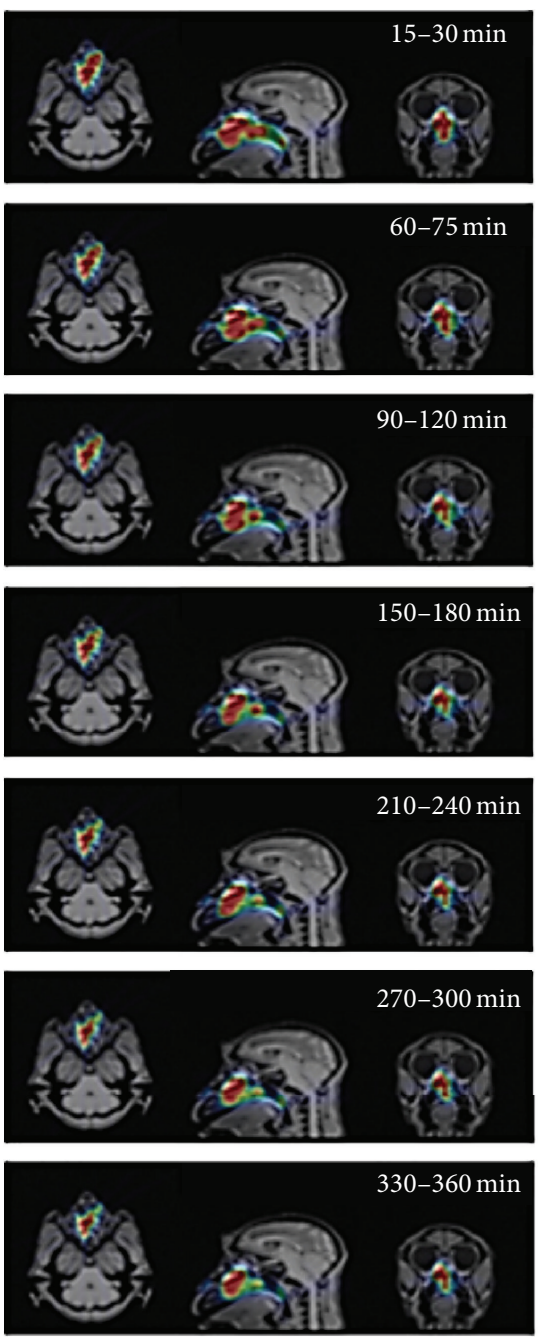

$\# 1^{\mathrm{a}}\left(\left[{ }^{18} \mathrm{~F}\right] \operatorname{Psp} A-\mathrm{PBS}\right)$
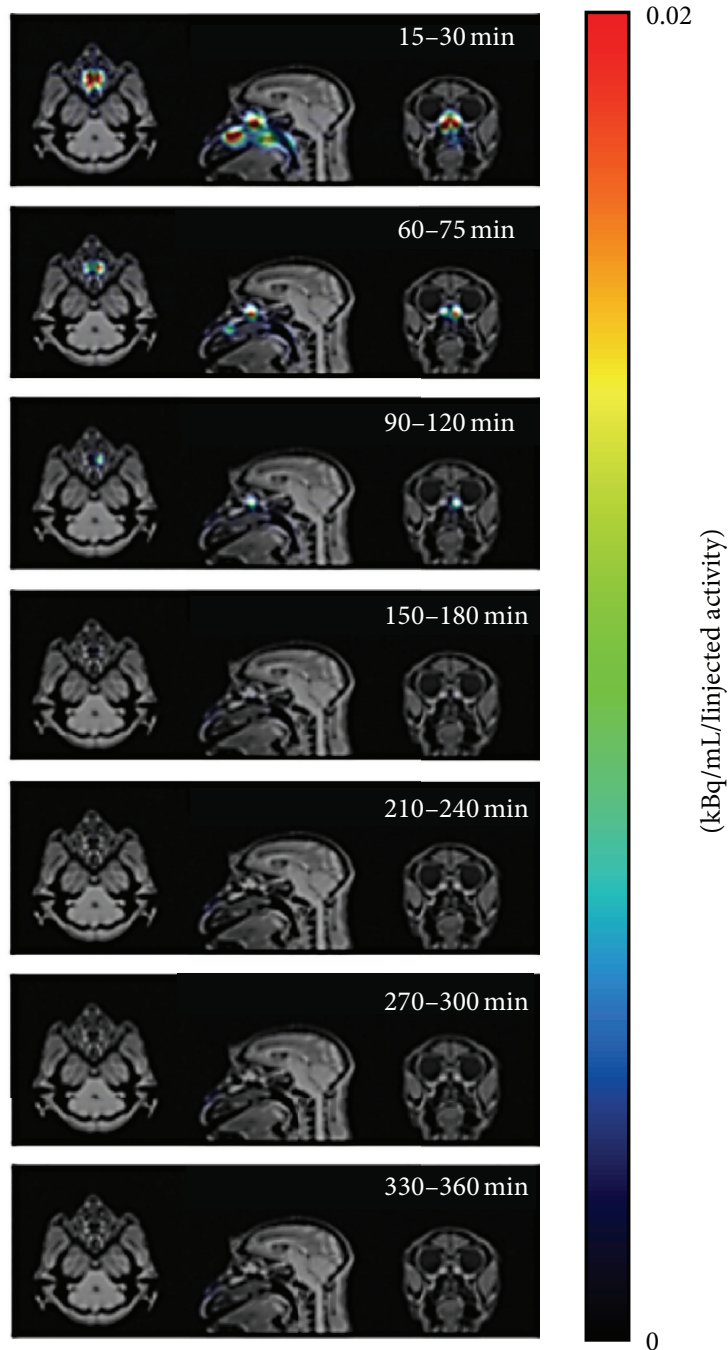

FIGURE 8: Positron emission tomography (PET) real-time images overlaid on MRI images are shown for the indicated times after nasal administration of $\left[{ }^{18} \mathrm{~F}\right]-P s p A$-nanogel or $\left[{ }^{18} \mathrm{~F}\right]-P s p A-P B S$ in a naive rhesus macaque. Adopted from Fukuyama et al. [115], with permission from Nature Publishing Group.

and efficiency as in vivo carrier transport. Nanogel carrier that could be conveniently used to target disease in most difficult locations such as those that could cross blood brain barrier is within human reach in the near future.

\section{Magnetic and Metallic Polymeric Nanomaterials}

The incorporation of superparamagnetic compounds into polymeric nanoparticles was reported to extend their potential applications to include contrast enhancement in magnetic resonance imaging (MRI), targeted drug delivery, hyperthermia, catalysis, biological separation, biosensors, and diagnostic medical devices [118]. Since the first report of historical application of magnetite grain in vivo as an antidote of accidental swallowing of rust by Avicenna [119], several magnetic polymeric nanoparticles were produced including those incorporating magnetite [120], maghemite [121], iron platinum [122], iron palladium [123], and iron oxide cobalt [124].

DNA vaccines are considered a cost-effective approach in managing and controlling infectious endemic diseases. Unfortunately, rapid in vivo degradation, short half-life of DNA, poor cell transfection, and lack of cell-specific targeting of the recent DNA vaccine prototypes present the biggest hurdle to the practical adoption of this therapeutic technique. The rate of DNA vaccine and siRNA delivery and cellular transfection were significantly improved by employing magnetically guided polymeric DNA nanocarriers termed "magnetofections" [125-129]. In order to trap DNAor drug-loaded magnetically probed nanocarriers, a strong external magnetic field is applied so that the nanocarriers are immobilized at the target tissue once delivered via the blood circulation. Thereafter, the DNA or drug is released 

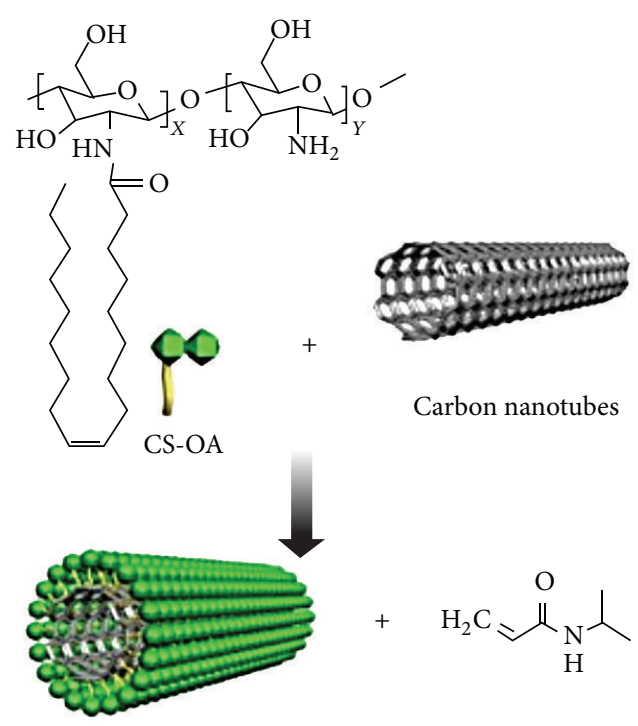

Carbon nanotubes

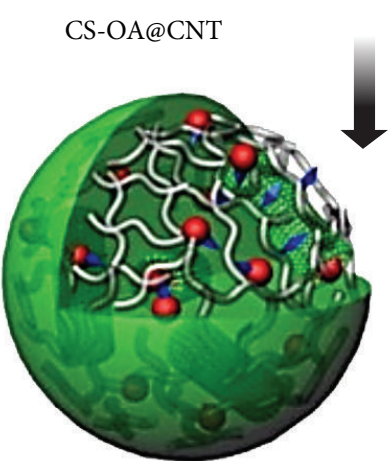

DOX loaded CS/PNIPAm@CNT
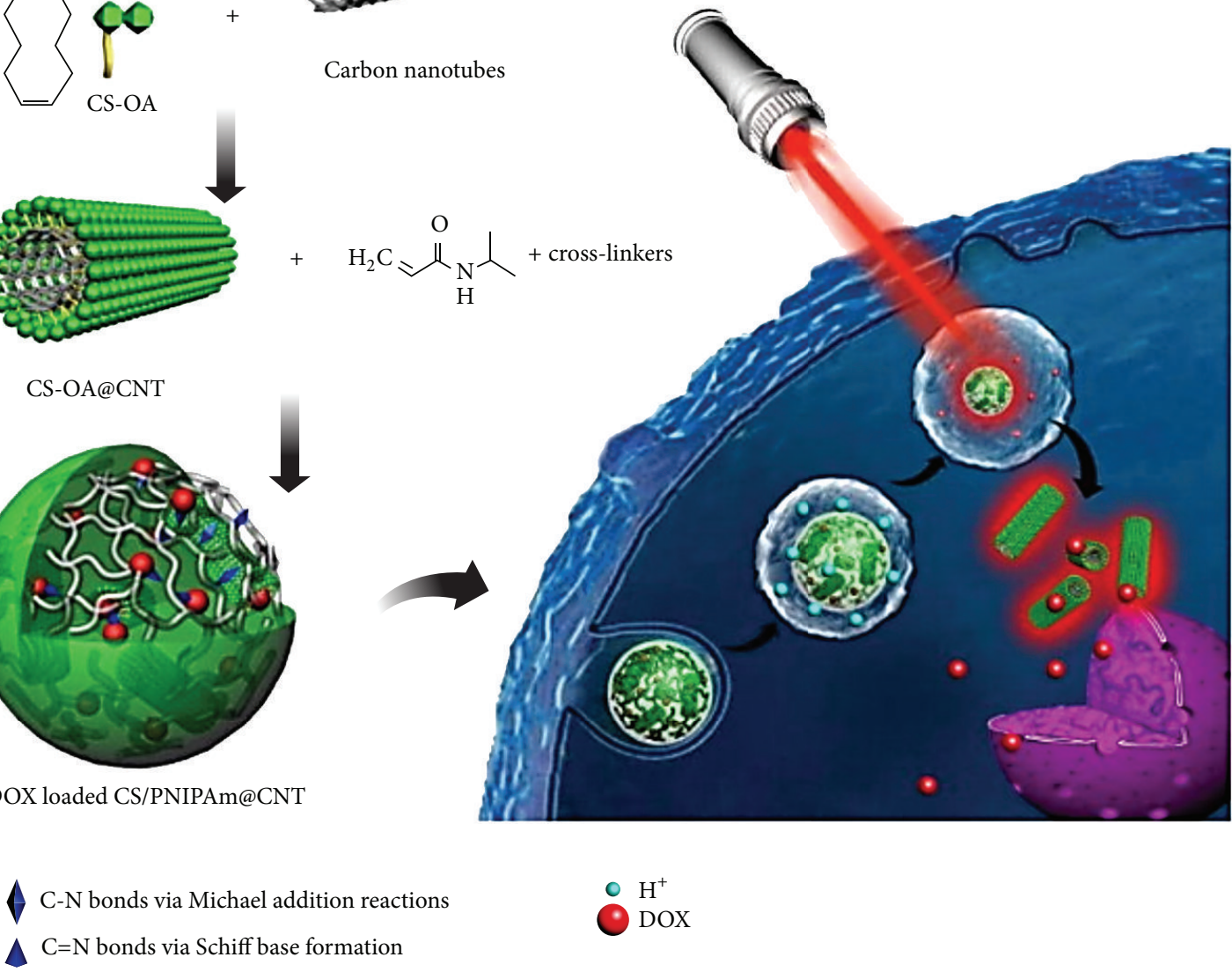

- $\mathrm{H}^{+}$

C DOX

FIGURE 9: Schematic illustration of near-infrared light activation of intracellular anticancer drug delivery using thermo-/pH sensitive polymeric nanoparticles (DOX/CS/PNIPAAm@CNT). Reprinted from Qin et al. [116], with permission from Elsevier.

from the carriers either by enzymatic degradation of the nanoparticle matrix or by charge interaction as reported previously [125]. The theoretical aspect of the extent of magnetic field to be applied externally to the body for the magnetic targeting has been studied extensively. It was shown that the efficiency of magnetic field to capture particles that are moving in blood vessels depends on several aspects such as blood flow rate and magnetic flux density. For example, it is shown that magnetic flux density of at least 200 to 700 millitesla (mT) and high field strength, namely, field gradients along the $z$-axis of 8 to $100 \mathrm{~T} / \mathrm{m}$, must be applied in order to effectively pull the particle out of suspension as they flow within the blood stream so that they could be entrapped or retained at the target tissue [125]. A magnetic flux density of $350 \mathrm{mT}$ was used by Chertok et al. [130] to investigate the efficiency of polyethyleneimine-modified iron oxide nanoparticles to deliver drug for brain tumor. Depending on the type of administration method used, the magnetic flux-assisted delivery could result in up to 30-fold increase in the entrapment of the nanoparticles towards the tumor.

Several methods for nanoparticle preparation were reported. These include (i) wet precipitation and coprecipitation that are based on manipulating the solution $\mathrm{pH}$ of the magnetic salt in such a way precipitation is favored; (ii) reverse-micelle mechanism that is based on the use of surfactant at critical micelle concentration in oil/organic solvent medium to trigger the self-assembly of the nanoparticles into micelles, and the final nanoparticle size is controlled by manipulating the precursor/surfactant ratio [124]; (iii) chemical vapor condensation, which involved heating the magnetic compound in inert atmosphere to form the nanoparticles; (iv) thermal decomposition and reduction that require the heating of metal oxides at elevated temperatures to decompose and reduce to nanoparticles; (v) liquid-phase reduction based on the principle of using a solution of 
strong reducing agent to help reduce the metallic oxide into magnetic metal nanoparticles. In all the methods, the magnetic nanoparticle serves as the core shell, where the polymeric matrix is then coated upon it through conjugation, layer-by-layer deposition, or microemulsion polymerization. McBain et al. [128] have elaborated on the methods of nanoparticles fabrication.

The surface modification of magnetic nanoparticle is of primary importance in extending its biomedical application. High surface-to-volume ratio and dipole-dipole interaction of the pristine magnetic nanoparticles have made them vulnerable to aggregation into large clusters culminating in colloidal instability and reduced intrinsic superparamagnetic properties [118]. The surface modification is necessary so as to make the magnetic nanoparticles more hydrophilic, biocompatible, and relatively easy to attach to the guiding probes for cellular tracking and specificity. For example, due to electrostatic interaction with the anionic surface of most cell membranes, nanoparticles with positively charged surface were more efficiently internalized in mammalian cells compared to nanoparticles that are negatively charged or with neutral surfaces. Several approaches related to nanoparticles surface modification have been investigated. Maghemite nanoparticles of about $29 \mathrm{~nm}$ were surface-modified by mixing them with protamine sulfate to endow a cationic surface charge properties on the maghemite particles [131]. This surface modification was reported to significantly enhance the in vitro cell culture transfection efficiency of hemagglutinating virus of Japan-envelope vector (HVJ-E), which rapidly transfers plasmid DNA, oligonucleotide, and protein into cells by cell-fusion. Similarly, polyethylenimine coated on magnetite $\left(\mathrm{Fe}_{3} \mathrm{O}_{4}\right)$ that was later conjugated with folic acid by ligand-conjugation method was developed resulting in magnetic nanoparticles (MNPs) with potential application in tumor targeting using MRI [132].

In another study, MNPs based on magnetite of $8 \mathrm{~nm}$ size were surface-modified by an in situ repetitive divergent polymerization using 3-aminopropyltrimethoxysilane (APTS), methyl acrylate, and ethylenediamine resulting in MNPs-functionalized polyamidoamine dendrimers (Table 1) that were used to efficiently deliver therapeutic gene to cancer cells [133]. The pristine magnetite was prepared by gradual addition of aqueous ammonia $(1.5 \mathrm{M})$ to a mixture of iron (III) chloride $(0.085 \mathrm{M})$ and iron (II) sulfate $(0.05 \mathrm{M})$ under nitrogen atmosphere and vigorous stirring, followed by ethanol washing, and recovered by applying external magnetic flux as indicated in the literature [134]. For surface modification, a portion of the pristine MNPs in ethanol $(150 \mathrm{~mL})$ was mixed with $10 \mathrm{~mL}$ of APTS after recovery. Subsequently, the coated MNPs were mixed with methyl acrylate followed by ethylenediamine to give the polyamidoamine dendrimers (Figure 10).

For in vivo applications of MNPs, especially as contrastenhancement aid during magnetic resonance imaging, the nanoparticles should have a long half-life for effective detection, diagnosis, and therapeutic management. There is the concern that the interaction between opsonin (a plasma base protein) and the MNPs would result in their accelerated clearance from blood circulation by macrophages phagocytosis
[118]. However, Mornet et al. [119] have shown that smaller particle size, particle surface neutrality, and hydrophilicity enhanced the plasma half-life of MNPs. Thus, MNPs surface modification using biocompatible hydrophilic polymers is an attractive proposition for the intended biomedical applications. For example, chitosan-coated MNPs efficiently produced high cellular imaging contrast and improved drug delivery characteristics have been reported $[135,136]$.

Recently, extended circulation time of MNPs capable of sustained tumor exposure through the circulation has been reported [137]. The study revealed the longevity and stability of the MNPs were incurred by coating with aminated cross-linked starch and subsequent modification with polyethylene glycol (PEG) using $\mathrm{N}$-Hydroxysuccinimide (NHS) chemistry. The study showed that MNPs modification using hydrophilic polymers resulted in about 10-fold less uptake by macrophages compared to the unmodified MNPs [137]. In addition, the modified MNPs also exhibited 150fold improvement in plasma stability and tumor exposure, where $24 \mathrm{~h}$ of sustained tumor exposure was observed [137]. Similarly, Larsen et al. [138] studied the effects of coating MNPs with PEG of different molecular weight $\left(M_{w}\right)$ (from 0.33 to $20 \mathrm{kDa}$ ) towards their physicochemical and biological properties. Using magnetic resonance imaging, they reported that in vivo uptake of PEG-coated MNPs in murine tumors is dependent upon the $M_{w}$ of PEG, with optimal $M_{w}$ at $10 \mathrm{kDa}$ [138]. The study also showed that PEG-coating at $M_{w}>5 \mathrm{kDa}$ helped to increase the hydrodynamic diameter of the MNPs, therefore resulting in reduced interaction with opsonin. This consequently prolonged the half-life of MNPs as their uptake by macrophages was significantly reduced [138]. Correspondingly, dextran-coated MNPs produced by coprecipitation method were observed to be highly stable in wide range of $\mathrm{pH}$ and $\mathrm{NaCl}$ concentrations, indicating their potential as yet another MRI contrast agent [139].

Osborne et al. [140] reported a novel microwave-assisted process for the production of dextran-coated MNPs. The coated MNPs possessed properties that are consistent for MRI contrast application. Drug delivery devices that integrate combined therapeutic and imaging diagnostic capabilities are termed "theranostics" and are highly sought after in cancer and other malignant disease therapies. Recently, low-frequency alternating magnetic field was used to study the controlled-release efficiency of theranostic magnetoliposomes nanoparticles. It is clear that coating the magnetoliposomes with carboxymethyl dextran enhanced both drug loading $(\cong 96.6 \%)$ and controlled release $(\cong 80 \%)$ efficiencies of the nanoparticles [15].

The biocompatibility and easiness of surface modification of gold nanoparticles (AuNPs) made them attractive for incorporation in biomedical and clinical applications [66]. Additionally, the optical properties of AuNPs following the excitation of plasmon oscillation, which causes the scattering and absorption of light at resonance wavelengths, are said to garner a lot of interest in terms of biomedical applications [141]. Taking the advantage of the glycol chitosan hydrophilicity, tumor targeting nanoparticles with high MRI contrast were produced by coating AuNPs with glycol chitosan [142]. In a similar study, gold(I) triphenylphosphine-conjugated 


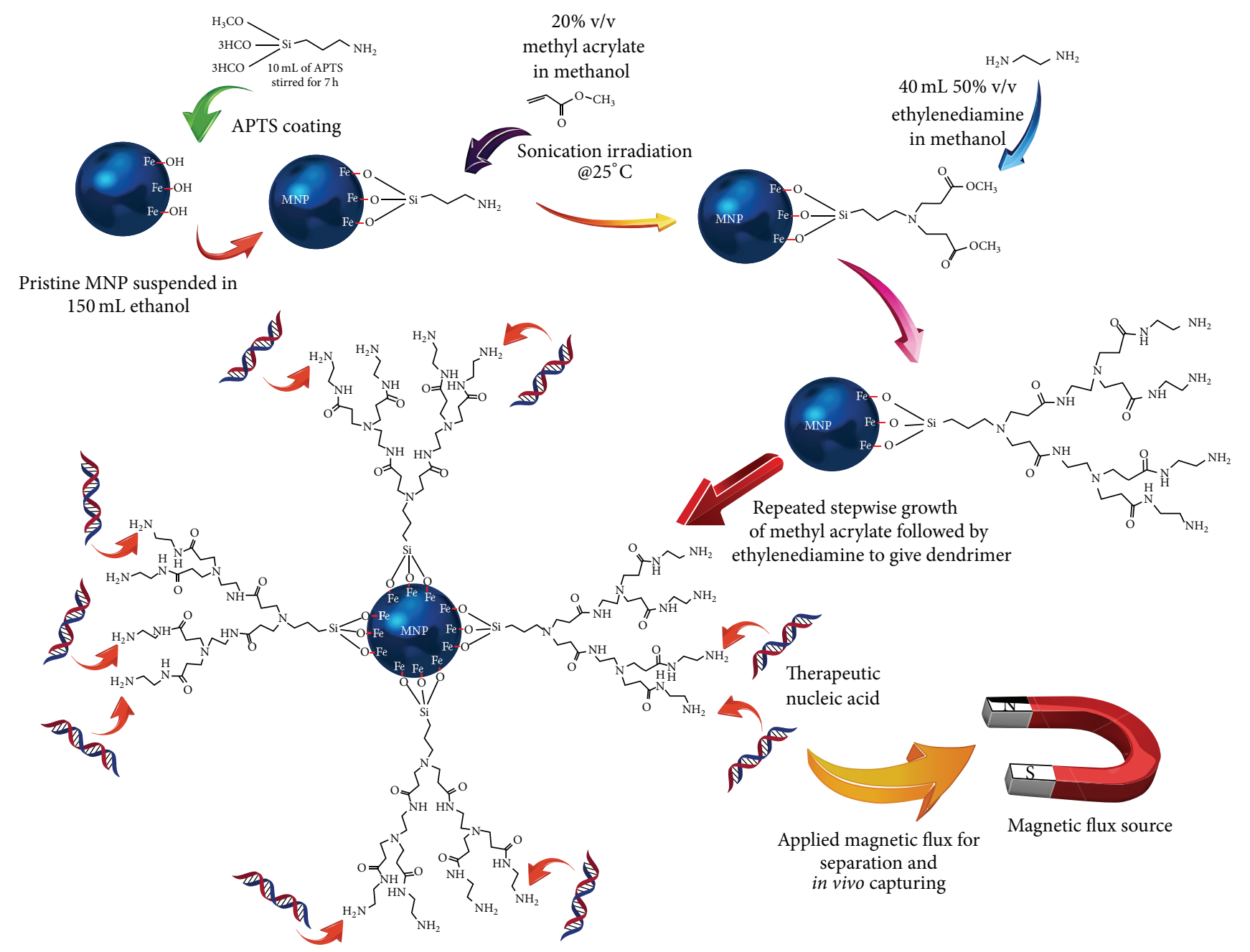

FIGURE 10: Schematic diagram of magnetic nanoparticle (MNP) surface modification and stepwise growth of dendrimer by in situ divergent polymerization.

glycopolymers nanoparticles were tested for their anticancer activity [143]. In another study, erythrocyte membranes coated gold nanocages were passively used in photothermal therapy for selective ablation of cancer cells in mouse model [144].

Previously, photosensitive gold nanocages coated with NIPAAm based smart polymer were prepared and tested as controlled drug carriers that are triggered to release the drug by near infrared laser [145]. The researchers further reported that combining the controlled release of the said smart polymer coated gold nanocages with the optical manipulation such as trapping resulted in an outstanding spatial/temporal resolution.

High-intensity focused ultrasound technique (HIFU) is a minimally invasive medical procedure that uses precise and focused ultrasound to rapidly heat and destroy pathogenic tissue [146]. In contrast to conventional radiation therapy, HIFU has no maximum cumulative dose and thus could be repeated until the tumor cells are destroyed [147]. Due to deep penetrating power of HIFU into soft tissues, this technique was tested for its potential application as an initiator for rapid on-demand drug release. In a recent study, the surface of gold nanocapsules was functionalized with LCST responsive poly(N-isopropylacrylamide) via gold-thiolate bonding and tested for HIFU triggered drug delivery application [148]. It was found that the polymer composite LCST can be tuned from 32 to $50^{\circ} \mathrm{C}$ to suit a number of applications simply by incorporating different concentrations of acrylamide [141]. Similarly, the antimicrobial activity of silver nanoparticles coated with bacterial cellulose and those conjugated with regenerated silk fibroin [149] was also reported. On the other hand, the efficacy of the antimicrobial activity of silver nanoparticles on silk fibroin degradation can be elucidated on other silk degrading bacteria genera like Bacillus, Pseudomonas, Streptomyces, and Serratia as well as fungal degrading silk fibroin [150].

Surface modification of magnetic nanoparticle with biopolymers has further extended their biomedical application mostly due to the enhanced biocompatibility subsequently improving the half-life of the MNPs in vivo. Although they have been proven to be effective against cancer cell line, it is however vital to advance the technique in order to pave 
the way for the preclinical trials of the anticancer fabricated drug delivery material in vivo. These will allow for extended application of MNPs.

\section{Concluding Remarks}

Biocompatible polymeric nanomaterials have garnered huge attention as potential vehicles for the delivery of chemotherapy drugs. Unfortunately, majority of the synthetic nanomaterials used in biomedical applications presented a number of deficiencies. For example, the nanomaterials used as drug carriers lack the ligand binding sites, responsiveness, and switchability to deliver the drugs to the target site and/or at the specified dosage and timing. Advancements in the fundamental understanding of supramolecular and chemistry of nanoconstruct system have led to the development of new synthetic approaches. They have made possible the fabrication of smart responsive polymeric nanomaterials of diversified applications within the biomedical research community.

The principle drawn upon biological inspiration is indispensable aid in designing nanomaterials that could translate, for example, chemical response(s) to easily interpretable information, thus making implementation of biosensors to become more effective tools and widely adopted. Timespecific and pulse-triggered drug released nanomaterials as well as self-healing and self-organized biomimetic nanomaterials to improve health management are fast becoming practical reality.

The current increasing demand of specialized medications, therapeutic devices, and smart medical procedures strongly drives the need for the fabrication and production of efficient nanoscale machinery that could closely match the natural systems. The increasing demand of noninvasive or mild-invasive therapies has favored the use of gene vaccines, DNAzymes, riboswitches, aptamers, and other polypeptides that need to be securely delivered to target site(s). The ability to manipulate nanoscale geometry and the incorporation of release trigger mechanism within the nanogel design undoubtedly will help to open up new frontier in drug delivery system. It brings the promise towards the improvement of drug loading capacity, efficient in vivo carrier transport, and the development of different stimuli guided devices, for example, polymer-conjugated magnetic or metallic nanoparticles, as drug delivery devices. Inadvertently, this expands the polymeric nanoparticle domain of applications beyond simple nanocarriers to theranostics capabilities. It is not unrealistic to anticipate that, in the near future, smart nanocarriers that could conveniently target diseases through some of the most inaccessible locations, such as blood brain barrier in order to efficiently manage Alzheimer's, Huntington's, and other neurodegenerative diseases, will be made available.

\section{Conflict of Interests}

The authors declare no conflict of interests, financially or otherwise.

\section{Acknowledgment}

The authors acknowledged University of Malaya for the research Grant BK014-2014.

\section{References}

[1] K. Bauri, S. G. Roy, S. Pant, and P. De, "Controlled synthesis of amino acid-based $\mathrm{pH}$-responsive chiral polymers and selfassembly of their block copolymers," Langmuir, vol. 29, no. 8, pp. 2764-2774, 2013.

[2] M. B. Dowling, J.-H. Lee, and S. R. Raghavan, "pH-responsive jello: gelatin gels containing fatty acid vesicles," Langmuir, vol. 25, no. 15, pp. 8519-8525, 2009.

[3] C. Galindo-Gonzalez, S. Gantz, L. Ourry, F. Mammeri, S. Ammar-Merah, and A. Ponton, "Elaboration and rheological investigation of magnetic sensitive nanocomposite biopolymer networks," Macromolecules, vol. 47, no. 9, pp. 3136-3144, 2014.

[4] W.-H. Hsieh, S.-F. Chang, H.-M. Chen, J.-H. Chen, and J. Liaw, "Oral gene delivery with cyclo-(d-Trp-Tyr) peptide nanotubes," Molecular Pharmaceutics, vol. 9, no. 5, pp. 1231-1249, 2012.

[5] E. Brewer, J. Coleman, and A. Lowman, "Emerging technologies of polymeric nanoparticles in cancer drug delivery," Journal of Nanomaterials, vol. 2011, Article ID 408675, 10 pages, 2011.

[6] K. Kim, M. Lee, H. Park et al., "Cell-permeable and biocompatible polymeric nanoparticles for apoptosis imaging," Journal of the American Chemical Society, vol. 128, no. 11, pp. 3490-3491, 2006.

[7] I. Roy and M. N. Gupta, "Smart polymeric materials: emerging biochemical applications," Chemistry \& Biology, vol. 10, no. 12, pp. 1161-1171, 2003.

[8] R. Cheng, F. Meng, C. Deng, H.-A. Klok, and Z. Zhong, "Dual and multi-stimuli responsive polymeric nanoparticles for programmed site-specific drug delivery," Biomaterials, vol. 34, no. 14 , pp. $3647-3657,2013$.

[9] C. C. Berry, S. Wells, S. Charles, and A. S. G. Curtis, "Dextran and albumin derivatised iron oxide nanoparticles: influence on fibroblasts in vitro," Biomaterials, vol. 24, no. 25, pp. 4551-4557, 2003.

[10] H.-J. Cho, I.-S. Yoon, H. Y. Yoon et al., "Polyethylene glycolconjugated hyaluronic acid-ceramide self-assembled nanoparticles for targeted delivery of doxorubicin," Biomaterials, vol. 33, no. 4, pp. 1190-1200, 2012.

[11] J. S. Rudra, S. Mishra, A. S. Chong et al., "Self-assembled peptide nanofibers raising durable antibody responses against a malaria epitope," Biomaterials, vol. 33, no. 27, pp. 6476-6484, 2012.

[12] R. Junka, C. M. Valmikinathan, D. M. Kalyon, and X. Yu, "Laminin functionalized biomimetic nanofibers for nerve tissue engineering," Journal of Biomaterials and Tissue Engineering, vol. 3, no. 4, pp. 494-502, 2013.

[13] A. M. Gumel and M. S. M. Annuar, "Nanocomposites of polyhydroxyalkanoates (PHAs)," in Polyhydroxyalkanoate (PHA) Based Blends, Composites and Nanocomposites, I. Roy and P. M. Visakh, Eds., pp. 98-118, Royal Society of Chemistry, London, UK, 2014.

[14] B. Pokroy, A. K. Epstein, M. C. M. Persson-Gulda, and J. Aizenberg, "Fabrication of bioinspired actuated nanostructures with arbitrary geometry and stiffness," Advanced Materials, vol. 21, no. 4, pp. 463-469, 2009. 
[15] H. Guo, W. Chen, X. Sun, Y.-N. Liu, J. Li, and J. Wang, "Theranostic magnetoliposomes coated by carboxymethyl dextran with controlled release by low-frequency alternating magnetic field," Carbohydrate Polymers, vol. 118, pp. 209-217, 2015.

[16] A. M. Gumel and M. S. M. Annuar, "Poly-3-hydroxyalkanoatesco-polyethylene glycol methacrylate copolymers for $\mathrm{pH}$ responsive and shape memory hydrogel," Journal of Applied Polymer Science, vol. 131, no. 23, 2014.

[17] W. E. Hennink, O. Franssen, W. N. E. van Dijk-Wolthuis, and H. Talsma, "Dextran hydrogels for the controlled release of proteins," Journal of Controlled Release, vol. 48, no. 2-3, pp. 107114, 1997.

[18] M. P. Fink, E. Abraham, J.-L. Vincent, and P. Kochanek, Textbook of Critical Care: With E-Edition, Saunders Elsevier, 2005.

[19] H. Beloeil, J.-X. Mazoit, D. Benhamou, and J. Duranteau, "Norepinephrine kinetics and dynamics in septic shock and trauma patients," British Journal of Anaesthesia, vol. 95, no. 6, pp. 782-788, 2005.

[20] A. M. Gumel, M. S. Annuar, K. A. Ishak, and N. Ahmad, "Carbon nanofibers-poly-3-hydroxyalkanoates nanocomposite: ultrasound-assisted dispersion and thermostructural properties," Journal of Nanomaterials, vol. 2014, Article ID 264206, 10 pages, 2014.

[21] K. Autumn, S. T. Hsieh, D. M. Dudek, J. Chen, C. Chitaphan, and R. J. Full, "Dynamics of geckos running vertically," Journal of Experimental Biology, vol. 209, no. 2, pp. 260-272, 2006.

[22] K. Autumn and A. M. Peattie, "Mechanisms of adhesion in geckos," Integrative and Comparative Biology, vol. 42, no. 6, pp. 1081-1090, 2002.

[23] M. J. McHenry and S. M. van Netten, "The flexural stiffness of superficial neuromasts in the zebrafish (Danio rerio) lateral line," The Journal of Experimental Biology, vol. 210, no. 23, pp. 4244-4253, 2007.

[24] P. A. Guerette, S. Hoon, Y. Seow et al., "Accelerating the design of biomimetic materials by integrating RNA-seq with proteomics and materials science," Nature Biotechnology, vol. 31, no. 10, pp. 908-915, 2013.

[25] A. J. Engler, S. Sen, H. L. Sweeney, and D. E. Discher, "Matrix elasticity directs stem cell lineage specification," Cell, vol. 126, no. 4, pp. 677-689, 2006.

[26] D. Discher, "Matrix elasticity directs stem cell lineage specification," APS March Meeting Abstracts, vol. 55, no. 2, p. 7003, 2010.

[27] J. T. Rutka, G. Apodaca, R. Stern, and M. Rosenblum, “The extracellular matrix of the central and peripheral nervous systems: structure and function," Journal of Neurosurgery, vol. 69, no. 2, pp. 155-170, 1988.

[28] S.-H. Chen, G.-J. Lai, and J.-P. Chen, "Preparation of biomimetic nanofibers by electrospinning of blends of silk fibroin and chitosan for bone tissue engineering," in Proceedings of the IEEE 4th International Nanoelectronics Conference (INEC '11), pp. 1-2, IEEE, Taoyuan, Taiwan, June 2011.

[29] S. Liao, B. Li, Z. Ma, H. Wei, C. Chan, and S. Ramakrishna, "Biomimetic electrospun nanofibers for tissue regeneration," Biomedical Materials, vol. 1, no. 3, p. R45, 2006.

[30] F. Wang, P. Liu, L. Sun, C. Li, V. A. Petrenko, and A. Liu, "Biomimetic nanostructure self-assembled from Au@Ag heterogeneous nanorods and phage fusion proteins for targeted tumor optical detection and photothermal therapy," Scientific Reports, vol. 4, article 6808, 2014.
[31] T.-S. Wong, S. H. Kang, S. K. Y. Tang et al., "Bioinspired selfrepairing slippery surfaces with pressure-stable omniphobicity," Nature, vol. 477, no. 7365, pp. 443-447, 2011.

[32] W. K. Laskey, C. W. Yancy, and W. H. Maisel, “Thrombosis in coronary drug-eluting stents: report from the meeting of the Circulatory System Medical Devices Advisory Panel of the Food and Drug Administration Center for Devices and Radiologic Health, December 7-8, 2006," Circulation, vol. 115, no. 17, pp. 2352-2357, 2007.

[33] D. C. Leslie, A. Waterhouse, J. B. Berthet et al., "A bioinspired omniphobic surface coating on medical devices prevents thrombosis and biofouling," Nature Biotechnology, vol. 32, no. 11, pp. 1134-1140, 2014.

[34] R. Hensel, A. Finn, R. Helbig et al., "Biologically inspired omniphobic surfaces by reverse imprint lithography," Advanced Materials, vol. 26, no. 13, pp. 2029-2033, 2014.

[35] A. Tuteja, W. Choi, J. M. Mabry, G. H. McKinley, and R. E. Cohen, "Robust omniphobic surfaces," Proceedings of the National Academy of Sciences of the United States of America, vol. 105, no. 47, pp. 18200-18205, 2008.

[36] P. Fuchs, C. Loeseken, J. K. Schubert, and W. Miekisch, "Breath gas aldehydes as biomarkers of lung cancer," International Journal of Cancer, vol. 126, no. 11, pp. 2663-2670, 2010.

[37] J. H. Lim, J. Park, E. H. Oh, H. J. Ko, S. Hong, and T. H. Park, "Nanovesicle-based bioelectronic nose for the diagnosis of lung cancer from human blood," Advanced Healthcare Materials, vol. 3, no. 3, pp. 360-366, 2014.

[38] Z. Ding, S. W. Annie Bligh, L. Tao et al., "Molecularly imprinted polymer based on MWCNT-QDs as fluorescent biomimetic sensor for specific recognition of target protein," Materials Science and Engineering C, vol. 48, pp. 469-479, 2015.

[39] L. P. Biró, "Photonic nanoarchitectures of biologic origin in butterflies and beetles," Materials Science and Engineering B, vol. 169, no. 1-3, pp. 3-11, 2010.

[40] L. P. Biró, K. Kertész, Z. Vértesy et al., "Living photonic crystals: butterfly scales-nanostructure and optical properties," Materials Science and Engineering C, vol. 27, no. 5-8, pp. 941-946, 2007.

[41] K. Kertész, G. Molnár, Z. Vértesy et al., "Photonic band gap materials in butterfly scales: a possible source of 'blueprints"' Materials Science and Engineering: B, vol. 149, no. 3, pp. 259265, 2008.

[42] E. Yablonovitch, "Inhibited spontaneous emission in solid-state physics and electronics," Physical Review Letters, vol. 58, no. 20, pp. 2059-2062, 1987.

[43] A. C. Arsenault, D. P. Puzzo, I. Manners, and G. A. Ozin, "Photonic-crystal full-colour displays," Nature Photonics, vol. 1, no. 8, pp. 468-472, 2007.

[44] K. M. Weeks and D. M. Mauger, "Exploring RNA structural codes with SHAPE chemistry," Accounts of Chemical Research, vol. 44, no. 12, pp. 1280-1291, 2011.

[45] W. W. Grabow and L. Jaeger, "RNA self-assembly and RNA nanotechnology," Accounts of Chemical Research, vol. 47, no. 6, pp. 1871-1880, 2014.

[46] S. Chakraborty, S. Mehtab, and Y. Krishnan, "The predictive power of synthetic nucleic acid technologies in RNA biology," Accounts of Chemical Research, vol. 47, no. 6, pp. 1710-1719, 2014.

[47] N. K. Navani and Y. Li, "Nucleic acid aptamers and enzymes as sensors," Current Opinion in Chemical Biology, vol. 10, no. 3, pp. 272-281, 2006. 
[48] K. Schlosser and Y. Li, "Biologically inspired synthetic enzymes made from DNA," Chemistry and Biology, vol. 16, no. 3, pp. 311$322,2009$.

[49] J. Li, C. Fan, H. Pei, J. Shi, and Q. Huang, "Smart drug delivery nanocarriers with self-assembled DNA nanostructures," Advanced Materials, vol. 25, no. 32, pp. 4386-4396, 2013.

[50] Y. Lu and J. Liu, "Smart nanomaterials inspired by biology: dynamic assembly of error-free nanomaterials in response to multiple chemical and biological stimuli," Accounts of Chemical Research, vol. 40, no. 5, pp. 315-323, 2007.

[51] J. C. Cochrane and S. A. Strobel, "Catalytic strategies of selfcleaving ribozymes," Accounts of Chemical Research, vol. 41, no. 8, pp. 1027-1035, 2008.

[52] R. Orbach, B. Willner, and I. Willner, "Catalytic nucleic acids (DNAzymes) as functional units for logic gates and computing circuits: from basic principles to practical applications," Chemical Communications, vol. 51, no. 20, pp. 4144-4160, 2015.

[53] I. Willner, B. Shlyahovsky, M. Zayats, and B. Willner, "DNAzymes for sensing, nanobiotechnology and logic gate applications," Chemical Society Reviews, vol. 37, no. 6, pp. 1153-1165, 2008.

[54] A. Haller, M. F. Soulière, and R. Micura, "The dynamic nature of RNA as key to understanding riboswitch mechanisms," Accounts of Chemical Research, vol. 44, no. 12, pp. 1339-1348, 2011.

[55] D. J. Goss and E. C. Theil, "Iron responsive mRNAs: a family of $\mathrm{Fe}^{2+}$ sensitive riboregulators," Accounts of Chemical Research, vol. 44, no. 12, pp. 1320-1328, 2011.

[56] E. G. Bellomo, M. D. Wyrsta, L. Pakstis, D. J. Pochan, and T. J. Deming, "Stimuli-responsive polypeptide vesicles by conformation-specific assembly," Nature Materials, vol. 3, no. 4, pp. 244-248, 2004.

[57] S. Zhang and Z. Li, "Stimuli-responsive polypeptide materials prepared by ring-opening polymerization of $\alpha$-amino acid $N$ carboxyanhydrides," Journal of Polymer Science B: Polymer Physics, vol. 51, no. 7, pp. 546-555, 2013.

[58] A. O. Elzoghby, W. M. Samy, and N. A. Elgindy, "Protein-based nanocarriers as promising drug and gene delivery systems," Journal of Controlled Release, vol. 161, no. 1, pp. 38-49, 2012.

[59] Q.-Y. Dong, M.-Y. Chen, Y. Xin et al., "Alginate-based and protein-based materials for probiotics encapsulation: a review," International Journal of Food Science and Technology, vol. 48, no. 7, pp. 1339-1351, 2013.

[60] A. M. Gumel, M. H. Aris, and M. S. M. Annuar, "Modification of polyhydroxyalkanoates (PHAs)," in Polyhydroxyalkanoate (PHA) Based Blends, Composites and Nanocomposites, I. Roy and P. M. Visakh, Eds., pp. 141-182, The Royal Society of Chemistry, London, UK, 2014.

[61] R. R. Breaker and G. F. Joyce, "A DNA enzyme that cleaves RNA," Chemistry \& Biology, vol. 1, no. 4, pp. 223-229, 1994.

[62] R. R. Breaker, "DNA enzymes," Nature Biotechnology, vol. 15, no. 5, pp. 427-431, 1997.

[63] Y. Tian and C. Mao, "DNAzyme amplification of molecular beacon signal," Talanta, vol. 67, no. 3, pp. 532-537, 2005.

[64] Y. Xiao, A. A. Rowe, and K. W. Plaxco, "Electrochemical detection of parts-per-billion lead via an electrode-bound DNAzyme assembly," Journal of the American Chemical Society, vol. 129, no. 2, pp. 262-263, 2007.

[65] X. Yang, J. Xu, X. Tang, H. Liu, and D. Tian, "A novel electrochemical DNAzyme sensor for the amplified detection of
$\mathrm{Pb}^{2+}$ ions," Chemical Communications, vol. 46, no. 18, pp. 31073109, 2010.

[66] P. M. Tiwari, K. Vig, V. A. Dennis, and S. R. Singh, "Functionalized gold nanoparticles and their biomedical applications," Nanomaterials, vol. 1, no. 1, pp. 31-63, 2011.

[67] K. Tram, P. Kanda, B. J. Salena, S. Huan, and Y. Li, "Translating bacterial detection by DNAzymes into a litmus test," Angewandte Chemie, vol. 126, no. 47, pp. 13013-13016, 2014.

[68] A. K. L. Darius, N. J. Ling, and U. Mahesh, "Visual detection of DNA from salmonella and mycobacterium using split DNAzymes," Molecular BioSystems, vol. 6, no. 5, pp. 792-794, 2010.

[69] C. W. Brown III, M. R. Lakin, D. Stefanovic, and S. W. Graves, "Catalytic molecular logic devices by DNAzyme displacement," ChemBioChem, vol. 15, no. 7, pp. 950-954, 2014.

[70] H. Gu, K. Furukawa, and R. R. Breaker, "Engineered allosteric ribozymes that sense the bacterial second messenger cyclic diguanosyl 5'-monophosphate," Analytical Chemistry, vol. 84, no. 11, pp. 4935-4941, 2012.

[71] K. A. Woodrow, Y. Cu, C. J. Booth, J. K. Saucier-Sawyer, M. J. Wood, and W. M. Saltzman, "Intravaginal gene silencing using biodegradable polymer nanoparticles densely loaded with small-interfering RNA," Nature Materials, vol. 8, no. 6, pp. 526533, 2009.

[72] K. Wang, Y. Li, G. Zhao et al., "Inhibition of human cytomegalovirus DNA replication by small interfering RNAs targeted to UL49," Acta Biochimica et Biophysica Sinica, vol. 45, no. 5, pp. 401-407, 2013.

[73] S. B. Kutluay, T. Zang, D. Blanco-Melo et al., "Global changes in the RNA binding specificity of HIV-1 Gag regulate virion genesis," Cell, vol. 159, no. 5, pp. 1096-1109, 2014.

[74] D. Ramalingam, S. Duclair, S. A. K. Datta, A. Ellington, A. Rein, and V. R. Prasad, "RNA aptamers directed to human immunodeficiency virus type 1 gag polyprotein bind to the matrix and nucleocapsid domains and inhibit virus production," Journal of Virology, vol. 85, no. 1, pp. 305-314, 2011.

[75] N. Sarver, E. M. Cantin, P. S. Chang et al., "Ribozymes as potential anti-HIV-1 therapeutic agents," Science, vol. 247, no. 4947, pp. 1222-1225, 1990.

[76] R. J. Scarborough, M. V. Lévesque, J.-P. Perreault, and A. Gatignol, "Design and evaluation of clinically relevant SOFA-HDV ribozymes targeting HIV RNA," in Therapeutic Applications of Ribozymes and Riboswitches, vol. 1103 of Methods in Molecular Biology, pp. 31-43, Springer, Berlin, Germany, 2014.

[77] J. Mulhbacher, P. St-Pierre, and D. A. Lafontaine, "Therapeutic applications of ribozymes and riboswitches," Current Opinion in Pharmacology, vol. 10, no. 5, pp. 551-556, 2010.

[78] P. He, D. Zhu, J.-J. Hu, J. Peng, L.-S. Chen, and G.-X. Lu, "PcDNA3.1(-)-mediated ribozyme targeting of HER-2 suppresses breast cancer tumor growth," Molecular Biology Reports, vol. 37, no. 3, pp. 1597-1604, 2010.

[79] K. E. Deigan and A. R. Ferré-D’Amaré, "Riboswitches: discovery of drugs that target bacterial gene-regulatory RNAs," Accounts of Chemical Research, vol. 44, no. 12, pp. 1329-1338, 2011.

[80] P. Ketzer, J. K. Kaufmann, S. Engelhardt et al., "Artificial riboswitches for gene expression and replication control of DNA and RNA viruses," Proceedings of the National Academy of Sciences of the United States of America, vol. 111, no. 5, pp. E554E562, 2014. 
[81] D. M. Floss, K. Schallau, S. Rose-John, U. Conrad, and J. Scheller, "Elastin-like polypeptides revolutionize recombinant protein expression and their biomedical application," Trends in Biotechnology, vol. 28, no. 1, pp. 37-45, 2010.

[82] S. R. MacEwan and A. Chilkoti, "Elastin-like polypeptides: biomedical applications of tunable biopolymers," Peptide Science, vol. 94, no. 1, pp. 60-77, 2010.

[83] J. Huang and A. Heise, "Stimuli responsive synthetic polypeptides derived from $\mathrm{N}$-carboxyanhydride (NCA) polymerisation," Chemical Society Reviews, vol. 42, no. 17, pp. 7373-7390, 2013.

[84] D. J. Callahan, W. Liu, X. Li et al., "Triple stimulus-responsive polypeptide nanoparticles that enhance intratumoral spatial distribution," Nano Letters, vol. 12, no. 4, pp. 2165-2170, 2012.

[85] K. E. Uhrich, S. M. Cannizzaro, R. S. Langer, and K. M. Shakesheff, "Polymeric systems for controlled drug release," Chemical Reviews, vol. 99, no. 11, pp. 3181-3198, 1999.

[86] A. C. R. Grayson, I. S. Choi, B. M. Tyler et al., "Multi-pulse drug delivery from a resorbable polymeric microchip device," Nature Materials, vol. 2, no. 11, pp. 767-772, 2003.

[87] A. J. Simnick, D. W. Lim, D. Chow, and A. Chilkoti, "Biomedical and biotechnological applications of elastin-like polypeptides," Polymer Reviews, vol. 47, no. 1, pp. 121-154, 2007.

[88] K. Wang, G.-F. Luo, Y. Liu et al., "Redox-sensitive shell crosslinked PEG-polypeptide hybrid micelles for controlled drug release," Polymer Chemistry, vol. 3, no. 4, pp. 1084-1090, 2012.

[89] G. Ciofani, G. G. Genchi, V. Mattoli, B. Mazzolai, and A. Bandiera, "The potential of recombinant human elastin-like polypeptides for drug delivery," Expert Opinion on Drug Delivery, vol. 11, no. 10, pp. 1507-1512, 2014.

[90] B. C. Dash, S. Mahor, O. Carroll et al., "Tunable elastin-like polypeptide hollow sphere as a high payload and controlled delivery gene depot," Journal of Controlled Release, vol. 152, no. 3, pp. 382-392, 2011.

[91] H.-C. Huang, C. R. Walker, A. Nanda, and K. Rege, "Laser welding of ruptured intestinal tissue using plasmonic polypeptide nanocomposite solders," ACS Nano, vol. 7, no. 4, pp. 2988-2998, 2013.

[92] C. M. Bellingham, M. A. Lillie, J. M. Gosline et al., "Recombinant human elastin polypeptides self-assemble into biomaterials with elastin-like properties," Biopolymers, vol. 70, no. 4, pp. 445-455, 2003.

[93] D. H. T. Le, R. Hanamura, D.-H. Pham et al., "Self-assembly of elastin-mimetic double hydrophobic polypeptides," Biomacromolecules, vol. 14, no. 4, pp. 1028-1034, 2013.

[94] L. Lorand and R. M. Graham, "Transglutaminases: crosslinking enzymes with pleiotropic functions," Nature Reviews Molecular Cell Biology, vol. 4, no. 2, pp. 140-156, 2003.

[95] M. K. McHale, L. A. Setton, and A. Chilkoti, "Synthesis and in vitro evaluation of enzymatically cross-linked elastin-like polypeptide gels for cartilaginous tissue repair," Tissue Engineering, vol. 11, no. 11-12, pp. 1768-1779, 2005.

[96] D. Bedell-Hogan, P. Trackman, W. Abrams, J. Rosenbloom, and H. Kagan, "Oxidation, cross-linking, and insolubilization of recombinant tropoelastin by purified lysyl oxidase," The Journal of Biological Chemistry, vol. 268, no. 14, pp. 10345-10350, 1993.

[97] U. M. Krishna, A. W. Martinez, J. M. Caves, and E. L. Chaikof, "Hydrazone self-crosslinking of multiphase elastin-like block copolymer networks," Acta Biomaterialia, vol. 8, no. 3, pp. 988997, 2012.
[98] S. Lv, M. Li, Z. Tang et al., "Doxorubicin-loaded amphiphilic polypeptide-based nanoparticles as an efficient drug delivery system for cancer therapy," Acta Biomaterialia, vol. 9, no. 12, pp. 9330-9342, 2013.

[99] X.-X. Xia, M. Wang, Y. Lin, Q. Xu, and D. L. Kaplan, "Hydrophobic drug-triggered self-assembly of nanoparticles from silkelastin-like protein polymers for drug delivery," Biomacromolecules, vol. 15, no. 3, pp. 908-914, 2014.

[100] W. Huang, A. Rollett, and D. L. Kaplan, "Silk-elastin-like protein biomaterials for the controlled delivery of therapeutics," Expert Opinion on Drug Delivery, vol. 12, no. 5, pp. 779-791, 2015.

[101] X. Huang, M. Li, D. C. Green, D. S. Williams, A. J. Patil, and S. Mann, "Interfacial assembly of protein-polymer nanoconjugates into stimulus-responsive biomimetic protocells," Nature Communications, vol. 4, article 2239, 2013.

[102] R. Yoshida, "Development of self-oscillating polymers and gels with autonomous function," Polymer Journal, vol. 42, no. 10, pp. 777-789, 2010.

[103] D. Suzuki, T. Sakai, and R. Yoshida, "Self-flocculating/selfdispersing oscillation of microgels," Angewandte Chemie, vol. 47, no. 5, pp. 917-920, 2008.

[104] Y. Sasaki and K. Akiyoshi, "Nanogel engineering for new nanobiomaterials: from chaperoning engineering to biomedical applications," Chemical Record, vol. 10, no. 6, pp. 366-376, 2010.

[105] N. Morimoto, X.-P. Qiu, F. M. Winnik, and K. Akiyoshi, "Dual stimuli-responsive nanogels by self-assembly of polysaccharides lightly grafted with thiol-terminated poly $(N$ isopropylacrylamide) chains," Macromolecules, vol. 41, no. 16, pp. 5985-5987, 2008.

[106] Y. Chen, X.-H. Pang, and C.-M. Dong, "Dual stimuli-responsive supramolecular polypeptidebased hydrogel and reverse micellar hydrogel mediated by host-guest chemistry," Advanced Functional Materials, vol. 20, no. 4, pp. 579-586, 2010.

[107] M. Oishi and Y. Nagasaki, "Synthesis, characterization, and biomedical applications of core-shell-type stimuliresponsive nanogels-nanogel composed of poly[2-(N,Ndiethylamino)ethyl methacrylate] core and PEG tethered chains," Reactive and Functional Polymers, vol. 67, no. 11, pp. 1311-1329, 2007.

[108] B. G. De Geest, C. Déjugnat, G. B. Sukhorukov, K. Braeckmans, S. C. De Smedt, and J. Demeester, "Self-rupturing microcapsules," Advanced Materials, vol. 17, no. 19, pp. 2357-2361, 2005.

[109] K. Raemdonck, B. Naeye, K. Buyens et al., "Biodegradable dextran nanogels for RNA interference: focusing on endosomal escape and intracellular siRNA delivery," Advanced Functional Materials, vol. 19, no. 9, pp. 1406-1415, 2009.

[110] R. Agarwal, V. Singh, P. Jurney, L. Shi, S. V. Sreenivasan, and K. Roy, "Mammalian cells preferentially internalize hydrogel nanodiscs over nanorods and use shape-specific uptake mechanisms," Proceedings of the National Academy of Sciences of the United States of America, vol. 110, no. 43, pp. 17247-17252, 2013.

[111] P. Cao, X. Sun, Y. Liang et al., "Gene delivery by a cationic and thermosensitive nanogel promoted established tumor growth inhibition," Nanomedicine, vol. 10, no. 10, pp. 1585-1597, 2015.

[112] L. C. Glangchai, M. Caldorera-Moore, L. Shi, and K. Roy, "Nanoimprint lithography based fabrication of shape-specific, enzymatically-triggered smart nanoparticles," Journal of Controlled Release, vol. 125, no. 3, pp. 263-272, 2008.

[113] J. A. Champion, Y. K. Katare, and S. Mitragotri, "Making polymeric micro- and nanoparticles of complex shapes," Proceedings 
of the National Academy of Sciences of the United States of America, vol. 104, no. 29, pp. 11901-11904, 2007.

[114] M. Toyoda, S. Hama, Y. Ikeda, Y. Nagasaki, and K. Kogure, "Anti-cancer vaccination by transdermal delivery of antigen peptide-loaded nanogels via iontophoresis," International Journal of Pharmaceutics, vol. 483, no. 1-2, pp. 110-114, 2015.

[115] Y. Fukuyama, Y. Yuki, Y. Katakai et al., "Nanogel-based pneumococcal surface protein A nasal vaccine induces microRNAassociated Th17 cell responses with neutralizing antibodies against Streptococcus pneumoniae in macaques," Mucosal Immunology, vol. 8, no. 5, pp. 1144-1153, 2015.

[116] Y. Qin, J. Chen, Y. Bi et al., "Near-infrared light remotecontrolled intracellular anti-cancer drug delivery using thermo/pH sensitive nanovehicle," Acta Biomaterialia, vol. 17, pp. 201-209, 2015.

[117] S. Lou, S. Gao, W. Wang et al., "Galactose-functionalized multiresponsive nanogels for hepatoma-targeted drug delivery," Nanoscale, vol. 7, no. 7, pp. 3137-3146, 2015.

[118] J. K. Oh and J. M. Park, "Iron oxide-based superparamagnetic polymeric nanomaterials: design, preparation, and biomedical application," Progress in Polymer Science, vol. 36, no. 1, pp. 168189, 2011.

[119] S. Mornet, S. Vasseur, F. Grasset, and E. Duguet, "Magnetic nanoparticle design for medical diagnosis and therapy," Journal of Materials Chemistry, vol. 14, no. 14, pp. 2161-2175, 2004.

[120] H. Ai, C. Flask, B. Weinberg et al., "Magnetite-loaded polymeric micelles as ultrasensitive magnetic-resonance probes," Advanced Materials, vol. 17, no. 16, pp. 1949-1952, 2005.

[121] A. F. Thünemann, D. Schütt, L. Kaufner, U. Pison, and H. Möhwald, "Maghemite nanoparticles protectively coated with poly(ethylene imine) and poly(ethylene oxide)-blockpoly(glutamic acid)," Langmuir, vol. 22, no. 5, pp. 2351-2357, 2006.

[122] S.-W. Chou, Y.-H. Shau, P.-C. Wu, Y.-S. Yang, D.-B. Shieh, and C.-C. Chen, "In vitro and in vivo studies of FePt nanoparticles for dual modal CT/MRI molecular imaging," Journal of the American Chemical Society, vol. 132, no. 38, pp. 13270-13278, 2010.

[123] R. S. Ningthoujam, R. K. Vatsa, A. Kumar, and B. N. Pandey, "Functionalized magnetic nanoparticles: concepts, synthesis and application in cancer hyperthermia," Functional Materials, pp. 229-260, 2012.

[124] T. E. Torres, A. G. Roca, M. P. Morales et al., "Magnetic properties and energy absorption of $\mathrm{CoFe}_{2} \mathrm{O}_{4}$ nanoparticles for magnetic hyperthermia," Journal of Physics: Conference Series, vol. 200, no. 7, Article ID 072101, 2010.

[125] J. Dobson, "Gene therapy progress and prospects: magnetic nanoparticle-based gene delivery," Gene Therapy, vol. 13, no. 4, pp. 283-287, 2006.

[126] T. J. Yoon, J. S. Kim, B. G. Kim, K. N. Yu, M. H. Cho, and J. K. Lee, "Multifunctional nanoparticles possessing a 'magnetic motor effect' for drug or gene delivery," Angewandte Chemie International Edition, vol. 117, no. 7, pp. 1092-1095, 2005.

[127] F. Scherer, M. Anton, U. Schillinger et al., "Magnetofection: enhancing and targeting gene delivery by magnetic force in vitro and in vivo," Gene Therapy, vol. 9, no. 2, pp. 102-109, 2002.

[128] S. C. McBain, H. H. P. Yiu, and J. Dobson, "Magnetic nanoparticles for gene and drug delivery," International Journal of Nanomedicine, vol. 3, no. 2, pp. 169-180, 2008.
[129] C. Plank, "Nanomedicine: silence the target," Nature Nanotechnology, vol. 4, no. 9, pp. 544-545, 2009.

[130] B. Chertok, A. E. David, and V. C. Yang, "Polyethyleneiminemodified iron oxide nanoparticles for brain tumor drug delivery using magnetic targeting and intra-carotid administration," Biomaterials, vol. 31, no. 24, pp. 6317-6324, 2010.

[131] N. Morishita, H. Nakagami, R. Morishita et al., "Magnetic nanoparticles with surface modification enhanced gene delivery of HVJ-E vector," Biochemical and Biophysical Research Communications, vol. 334, no. 4, pp. 1121-1126, 2005.

[132] L. Li, F. Gao, W. Jiang et al., "Folic acid-conjugated superparamagnetic iron oxide nanoparticles for tumor-targeting MR imaging," Drug Delivery, 2015.

[133] B. Pan, D. Cui, Y. Sheng et al., "Dendrimer-modified magnetic nanoparticles enhance efficiency of gene delivery system," Cancer Research, vol. 67, no. 17, pp. 8156-8163, 2007.

[134] B.-F. Pan, F. Gao, and H.-C. Gu, "Dendrimer modified magnetite nanoparticles for protein immobilization," Journal of Colloid and Interface Science, vol. 284, no. 1, pp. 1-6, 2005.

[135] Y. Ge, Y. Zhang, S. He, F. Nie, G. Teng, and N. Gu, "Fluorescence modified chitosan-coated magnetic nanoparticles for highefficient cellular imaging," Nanoscale Research Letters, vol. 4, no. 4, pp. 287-295, 2009.

[136] L. Zhu, J. Ma, N. Jia, Y. Zhao, and H. Shen, "Chitosancoated magnetic nanoparticles as carriers of 5-fluorouracil: preparation, characterization and cytotoxicity studies," Colloids and Surfaces B: Biointerfaces, vol. 68, no. 1, pp. 1-6, 2009.

[137] A. J. Cole, A. E. David, J. Wang, C. J. Galbán, H. L. Hill, and V. C. Yang, "Polyethylene glycol modified, cross-linked starchcoated iron oxide nanoparticles for enhanced magnetic tumor targeting," Biomaterials, vol. 32, no. 8, pp. 2183-2193, 2011.

[138] E. K. U. Larsen, T. Nielsen, T. Wittenborn et al., "Accumulation of magnetic iron oxide nanoparticles coated with variably sized polyethylene glycol in murine tumors," Nanoscale, vol. 4, no. 7, pp. 2352-2361, 2012.

[139] G. Liu, R. Y. Hong, L. Guo, Y. G. Li, and H. Z. Li, "Preparation, characterization and MRI application of carboxymethyl dextran coated magnetic nanoparticles," Applied Surface Science, vol. 257, no. 15, pp. 6711-6717, 2011.

[140] E. A. Osborne, T. M. Atkins, D. A. Gilbert, S. M. Kauzlarich, K. Liu, and A. Y. Louie, "Rapid microwave-assisted synthesis of dextran-coated iron oxide nanoparticles for magnetic resonance imaging," Nanotechnology, vol. 23, no. 21, Article ID 215602, 2012.

[141] Y. Xia, W. Li, C. M. Cobley et al., "Gold nanocages: from synthesis to theranostic applications," Accounts of Chemical Research, vol. 44, no. 10, pp. 914-924, 2011.

[142] I.-C. Sun, J. H. Na, S. Y. Jeong et al., "Biocompatible glycol chitosan-coated gold nanoparticles for tumor-targeting CT imaging," Pharmaceutical Research, vol. 31, no. 6, pp. 1418-1425, 2014.

[143] C. K. Adokoh, S. Quan, M. Hitt, J. Darkwa, P. Kumar, and R. Narain, "Synthesis and evaluation of glycopolymeric decorated gold nanoparticles functionalized with gold-triphenyl phosphine as anti-cancer agents," Biomacromolecules, vol. 15, no. 10, pp. 3802-3810, 2014.

[144] J.-G. Piao, L. Wang, F. Gao, Y.-Z. You, Y. Xiong, and L. Yang, "Erythrocyte membrane is an alternative coating to polyethylene glycol for prolonging the circulation lifetime of gold nanocages for photothermal therapy," ACS Nano, vol. 8, no. 10, pp. 10414-10425, 2014. 
[145] M. S. Yavuz, Y. Cheng, J. Chen et al., "Gold nanocages covered by smart polymers for controlled release with near-infrared light," Nature Materials, vol. 8, no. 12, pp. 935-939, 2009.

[146] J. E. Kennedy, G. R. Ter Haar, and D. Cranston, "High intensity focused ultrasound: surgery of the future?" The British Journal of Radiology, vol. 76, no. 909, pp. 590-599, 2003.

[147] G. ter Haar and C. Coussios, "High intensity focused ultrasound: physical principles and devices," International Journal of Hyperthermia, vol. 23, no. 2, pp. 89-104, 2007.

[148] W. Li, X. Cai, C. Kim et al., "Gold nanocages covered with thermally-responsive polymers for controlled release by highintensity focused ultrasound," Nanoscale, vol. 3, no. 4, pp. 17241730, 2011.

[149] Z. Lu, M. Meng, Y. Jiang, and J. Xie, "UV-assisted in situ synthesis of silver nanoparticles on silk fibers for antibacterial applications," Colloids and Surfaces A: Physicochemical and Engineering Aspects, vol. 447, pp. 1-7, 2014.

[150] B. Gutarowska and A. Michalski, "Microbial degradation of woven fabrics and protection against biodegradation," in Woven Fabrics, H.-Y. Jeon, Ed., chapter 10, InTech, Rijeka, Croatia, 2012. 

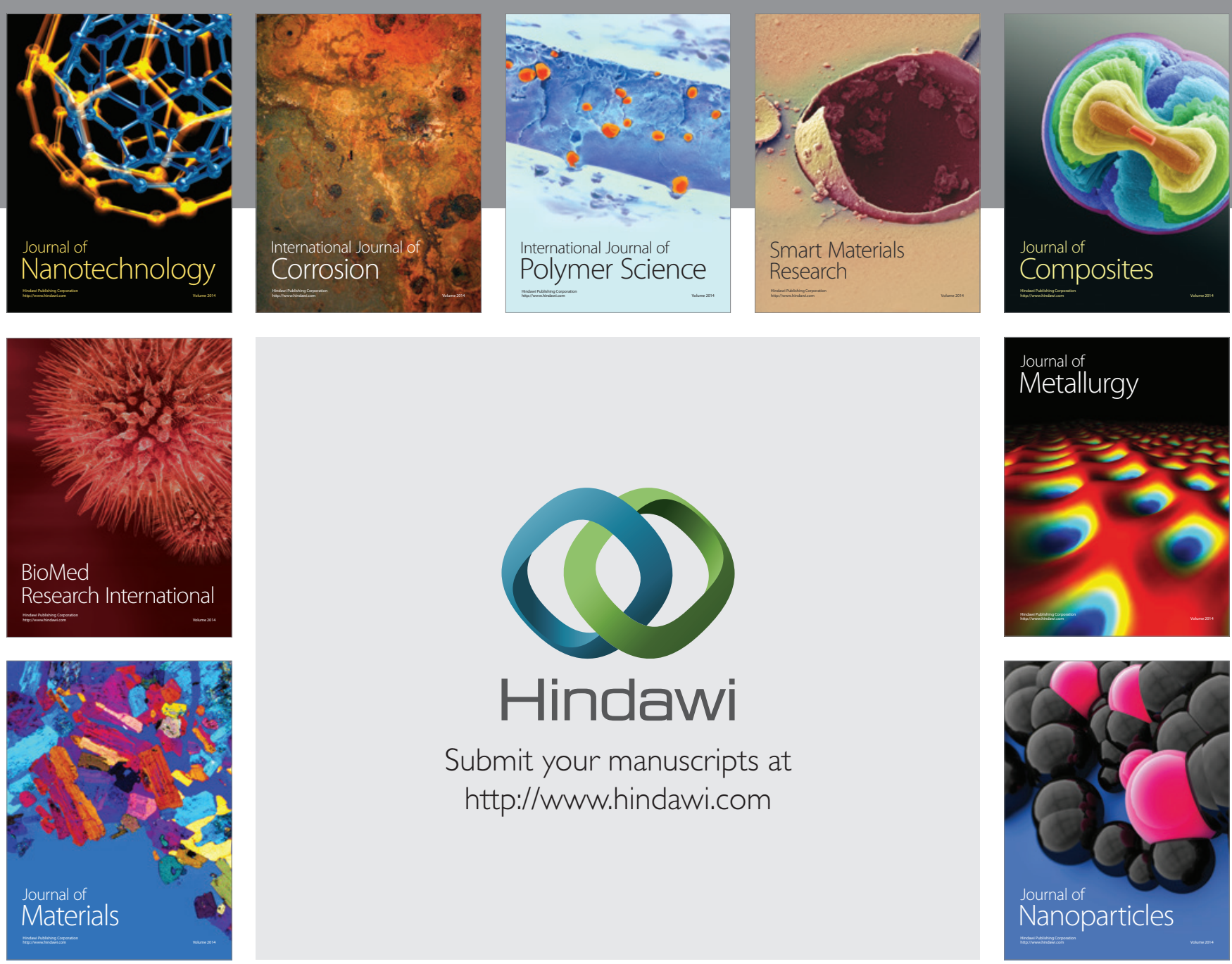

Submit your manuscripts at http://www.hindawi.com
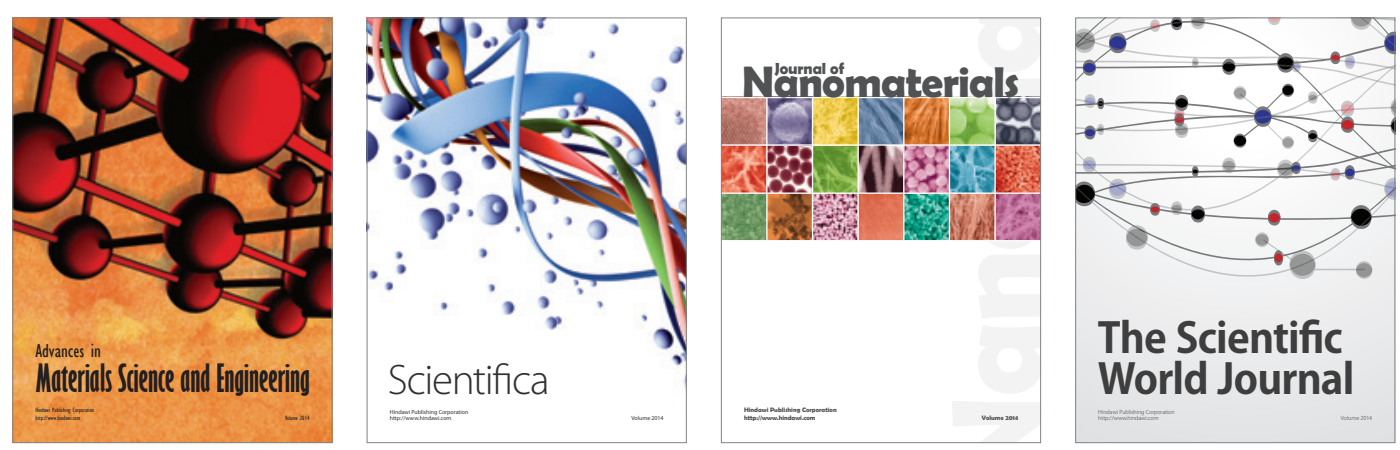

\section{The Scientific World Journal}
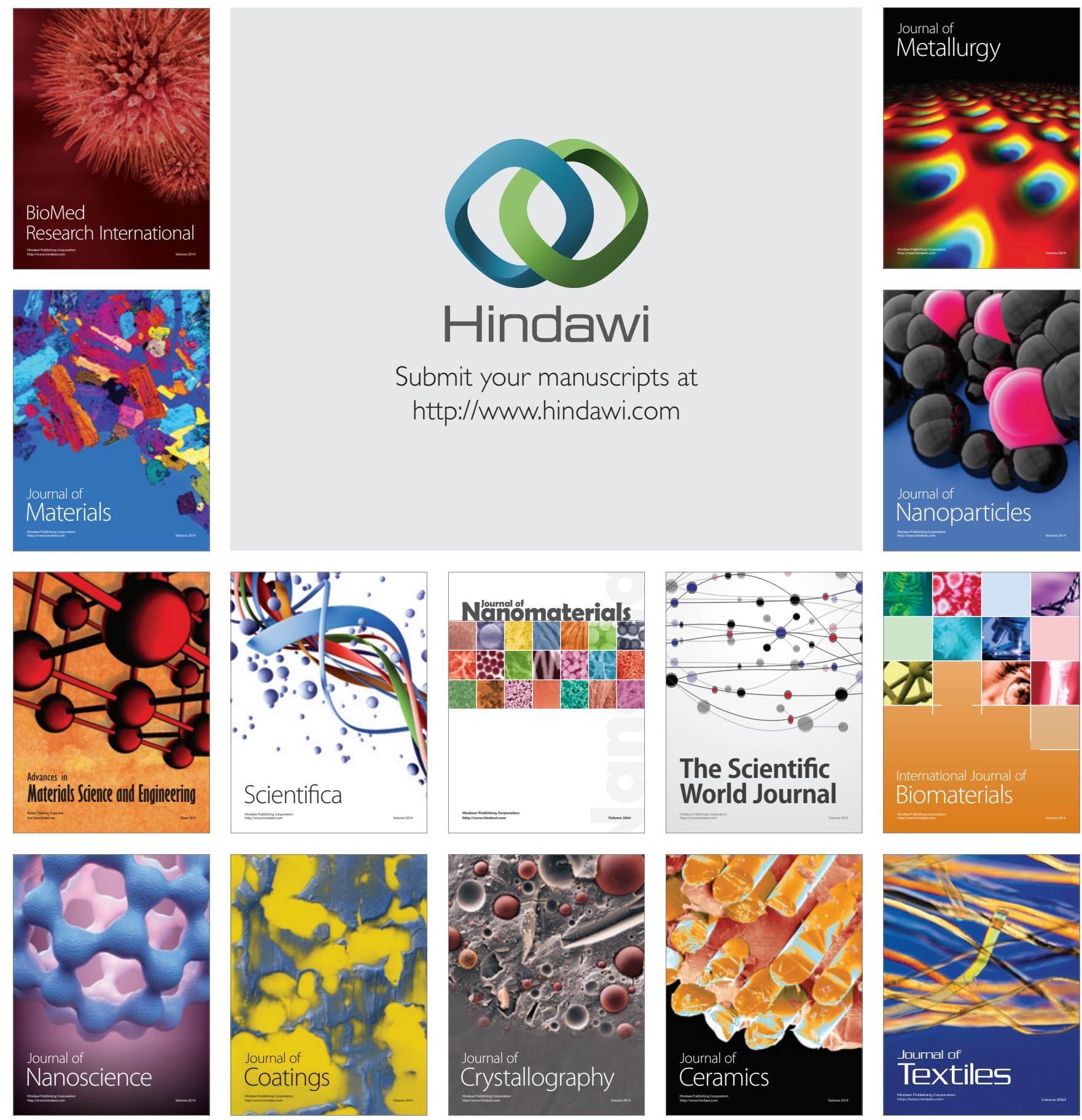